\title{
TRANSVERSE INVARIANTS AND EXOTIC SURFACES IN THE 4-BALL
}

\author{
ANDRÁS JUHÁSZ, MAGGIE MILLER, AND IAN ZEMKE
}

\begin{abstract}
Using 1-twist rim surgery, we construct infinitely many smoothly embedded, orientable surfaces in the 4-ball bounding a knot in the 3-sphere that are pairwise topologically isotopic, but not ambient diffeomorphic. We distinguish the surfaces using the maps they induce on perturbed link Floer homology. Along the way, we show that the cobordism map induced by an ascending surface in a Weinstein cobordism preserves the transverse invariant in link Floer homology.
\end{abstract}

\section{INTRODUCTION}

Let $S$ be a smooth surface in the smooth 4 -manifold $X$. Then we say that the surface $S^{\prime}$ is an exotic copy of $S$ if $S$ and $S^{\prime}$ are topologically isotopic, but not smoothly isotopic. It is a fundamental open conjecture in the theory of knotted surfaces that there are no exotic orientable unknots of any genus in $S^{4}$. In fact, currently there is no example of any exotic pair of closed oriented surfaces in $S^{4}$. Note that, in the non-orientable case, Finashin, Kreck, and Viro [FKV87] constructed an infinite family of exotic copies of the standardly embedded $\#_{10} \mathbb{R} P^{2}$ in $S^{4}$. We provide the first examples of exotic orientable surfaces in the 4-ball, and in fact distinguish them up to diffeomorphism (that is not necessarily the identity on $S^{3}$ ) using perturbed cobordism maps on link Floer homology. We note that Gompf [Gom91, Theorem 8.1] constructed punctured tori in $B^{4}$ that are non-diffeomorphic, and he conjectured to be pairwise homeomorphic.

Theorem 1.1. There are infinitely many knots in $S^{3}$ such that each bounds countably infinitely many properly embedded, smooth, orientable, genus one surfaces in $B^{4}$ that are pairwise topologically isotopic, but there is no diffeomorphism of $B^{4}$ taking one to the other.

Exotic pairs of orientable surfaces in other 4-manifolds are constructed using variations of the rim surgery operation of Fintushel and Stern [FS97]. For example, Finashin [Fin02] constructed smoothly inequivalent surfaces in $\mathbb{C} P^{2}$, which Kim and Ruberman proved to be topologically isotopic [KR08a]. To show topological isotopy, previous constructions require the surface complement to be simply-connected, or at least to have finite cyclic fundamental group; see Kim and Ruberman [KR08a][KR08b]. The surfaces are distinguished using Seiberg-Witten invariants. It is unclear if these methods give rise to exotic surfaces in $S^{4}$.

In Theorem 2.5, we provide a method for constructing surfaces that are topologically isotopic (and are hence potentially exotic), where the fundamental group of the surface complement is irrelevant. We use twist rim surgery, introduced by Kim [Kim06a]. This combines the twist-spinning construction of 2-knots, due to Zeeman [Zee65], with rim surgery. Zeeman showed that a 1-twistspun 2-knot is always smoothly trivial. This can be rephrased as follows: 1-twist rim surgery on an unknotted 2-sphere in $S^{4}$ is smoothly trivial. Building on this result, in Section 2.2, we show that, if $S$ is a surface in a 4-manifold, and $\gamma \subseteq S$ is a simple closed curve that bounds a topologically embedded disk $D$ in the complement of $S$, then the result $S^{\prime}$ of 1-twist rim surgery on $S$ along $\gamma$ is topologically isotopic to $S$. When $D$ is smooth, then $S^{\prime}$ is smoothly isotopic to $S$. In fact, our result holds for any concordance rim surgery - a generalization of twist rim surgery that we introduce in Section 2.1 - that gives the unknotted 2-sphere when performed along the equator of $S^{2} \subseteq S^{4}$. Note that Kim and Ruberman [KR08a, Corollary 4.6] gave a different proof of Theorem 2.5 in the case of 1-twist rim surgery that relies on the 4-dimensional topological $s$-cobordism theorem, assuming

2010 Mathematics Subject Classification. 57R58; 57R55; 57M27.

Key words and phrases. 4-manifolds, exotic surfaces, Heegaard Floer homology. 
the fundamental group of the complement of $S$ is good in the sense of Freedman, and that the knot used for the rim surgery is slice.

Hence, if we find a pair $(S, \gamma)$, where $\gamma$ bounds a topological disk, but not a smooth disk, in the complement of $S$, then we can construct infinitely many potentially exotic copies of $S$. This is the case whenever $S$ is a Seifert surface in $S^{3}$ pushed into $B^{4}$, and $\gamma \subseteq S$ comes from a non-separating, Alexander polynomial one knot on the Seifert surface with trivial surface framing. We perform 1-twist rim surgery on this pair $(S, \gamma)$. (Note that $\pi_{1}\left(B^{4} \backslash S\right) \cong \mathbb{Z}$, since $S$ comes from $S^{3}$.)

What remains is to show that the resulting surfaces are not diffeomorphic. For this, we use the cobordism maps induced by the surfaces on perturbed link Floer homology. The effect of concordance rim surgery on these maps follows from the work of the first and third authors [JZ18a]; see Theorem 5.1 for the precise formula.

To distinguish the maps, we need that the map induced by $S$ is non-vanishing, that $\gamma$ is homologically non-trivial on $S$, and that the pattern we use for the 1-twist rim surgery has non-trivial Alexander polynomial; see Theorem 6.6. We achieve the first condition by finding a quasipositive $S$, and showing that such surfaces induce non-vanishing maps on link Floer homology, as they preserve the transverse invariant in knot Floer homology defined by Lisca, Ozsváth, Stipsicz, and Szabó [LOSS09], and extended by Baldwin, Vela-Vick, and Vértesi [BVVV13] (usually referred to as the LOSS or BRAID invariant); see Corollary 8.3. For example, $S$ can be the standard genus one Seifert surface of a twice iterated, positive, untwisted Whitehead double of any nontrivial, strongly quasipositive knot.

In fact, Corollary 8.3 is a special case of the following much more general result:

Theorem 1.2. Suppose $(W, \mathcal{S}):\left(Y_{0}, \mathbb{L}_{0}\right) \rightarrow\left(Y_{1}, \mathbb{L}_{1}\right)$ is a decorated link cobordism such that $W$ has a Weinstein structure $(W, \omega, \phi, V)$, and $\mathcal{S}=(S, \mathcal{A})$, where $S$ is an ascending surface with positive critical points. If the decoration $\mathcal{A}$ is $\mathbf{w}$-anti-arboreal with respect to $\phi$, then

$$
\left(F_{W, \mathcal{S}}^{\circ}\right)^{\vee}\left(\mathfrak{T}^{\circ}\left(\mathbb{L}_{1}\right)\right)=\mathfrak{T}^{\circ}\left(\mathbb{L}_{0}\right)
$$

for $\circ \in\{\wedge,-\}$, where $\mathfrak{T}^{\circ}\left(\mathbb{L}_{i}\right)$ is the transverse invariant of the transverse link $\mathbb{L}_{i}$ for $i \in\{0,1\}$.

See Section 7 for some background on ascending surfaces in Weinstein cobordisms, and Definition 8.1 for $\mathbf{w}$-anti-arboreal decorations. We prove Theorem 1.2 in Section 8. Theorem 1.2 fits into the context of a family of similar results due to Ozsváth and Szabó [OS05, Theorem 1.5], the first author [Juh16, Theorem 11.24], Baldwin and Sivek [BS16, Theorem 1.10] [BS18, Theorem 1.2], Golla and the first author [GJ19], Baldwin, Lidman, and Wong [BLW19, Theorem 1.5], and Kang [Kan18a][Kan18b].

To distinguish the surfaces up to diffeomorphism using the knot Floer cobordism maps, we define an invariant $\Omega(S) \in \mathbb{Z}^{\geq 0} \cup\{-\infty\}$ for any orientable surface of genus $g>0$ bounding a knot $K$ in $S^{3}$. We prove that $\Omega(S)$ is an invariant of the diffeomorphism type of the pair $\left(B^{4}, S\right)$ in Theorem 6.3. Furthermore, if $S$ is a surface bounding $K$, and $S^{\prime}$ is obtained by 1-twist rim surgery using an auxiliary knot $J$ in $S^{3}$, we prove that

$$
\Omega\left(S^{\prime}\right)=\Omega(S)+\operatorname{Irr}\left(\Delta_{J}(t)\right)
$$

where $\operatorname{Irr}\left(\Delta_{J}(t)\right)$ is the number of irreducible factors of the Alexander polynomial of $J$. Finally, if $S$ is a quasipositive surface in $B^{4}$, we prove that

$$
\Omega(S)=0
$$

see Proposition 8.5. In fact, an algebraic argument of Sunukjian [Sun15, Theorem 2], combined with Theorem 1.2, implies that $S$ and $S^{\prime}$ are never diffeomorphic whenever $S$ is a quasipositive surface and $\Delta_{J}(t) \neq 1$, though this is not immediately sufficient for Theorem 1.1.

Instead of the link cobordism maps, one could distinguish the surfaces we construct up to diffeomorphism fixing $S^{3}$ pointwise using Seiberg-Witten theory, as follows: By Rudolph [Rud83], every quasipositive surface $S$ in $B^{4}$ is algebraic. Hence, we can consider the projectivization $\hat{S}$ of $S$ in $\mathbb{C P}^{2}$, and perturb it to be nonsingular. Then one can apply the result of Kim [Kim06b, Theorem 3.4] to compute the effect of twist rim surgery on the relative Seiberg-Witten invariant $\mathrm{SW}_{\mathbb{C P}^{2}, \hat{S}}$ of the 
complement of $\hat{S}$. Note that this method does not extend to general concordance surgeries, due to the lack of a concordance surgery formula on the Seiberg-Witten side.

It is a natural question whether one can use Theorem 1.1 to obtain an exotic pair of orientable closed surfaces in $S^{4}$. A natural candidate would be obtained by taking the surfaces $S$ and $S^{\prime}$, constructed in Theorem 1.1, and considering $S \cup-S^{\prime} \subseteq S^{4}$. However, it is a straightforward exercise to see that this surface is smoothly unknotted.

1.1. Acknowledgements. We would like to thank Peter Feller for his invaluable guidance on quasipositive surfaces. We would also like to thank Anthony Conway, Robert Gompf, Kyle Hayden, Hee Jun Kim, Anubhav Mukherjee, Mark Powell, and Daniel Ruberman for helpful discussions.

The first author was supported by a Royal Society Research Fellowship, the second author by an NSF Graduate Research Fellowship (DGE-1656466), and the third author by an NSF Postdoctoral Research Fellowship (DMS-1703685). This project has received funding from the European Research Council (ERC) under the European Union's Horizon 2020 research and innovation programme (grant agreement No 674978).

\section{Concordance Rim SURgery and topological isotopy}

2.1. Concordance rim surgery. Concordance surgery is a generalization of knot surgery introduced by Fintushel and Stern; see Akbulut [Akb01] and the work of the first and third authors [JZ18a]. In this section, we present a generalization of rim surgery, based on concordance surgery.

Suppose $S$ is a properly embedded, smooth, oriented surface in the smooth, compact 4-manifold $W$, and $\gamma$ is a simple closed curve in $S$. We identify neighborhood of $\gamma$ in $(W, S)$ with $\left(S^{1} \times B^{3}, S^{1} \times a\right)$, where $a \subseteq B^{3}$ denotes a properly embedded, unknotted arc.

Definition 2.1. Suppose $S$ is a properly embedded, smooth, oriented surface in the compact 4manifold $W$, and $\gamma \subseteq S$ is a simple closed curve. Furthermore, let $\left(I \times S^{3}, C\right)$ be a self-concordance of a knot $K \subseteq S^{3}$. We assume that there is a neighborhood $N(p) \subseteq S^{3}$ of a point $p \in K$ such that the $\operatorname{arc} N(p) \cap K$ is unknotted, and

$$
(I \times N(p)) \cap C=I \times(N(p) \cap K) .
$$

If we glue $\{0\} \times S^{3}$ and $\{1\} \times S^{3}$ using the equivalence relation $(0, x) \sim(1, x)$ for $x \in S^{3}$, then we obtain the annulus $A:=(C \backslash(I \times N(p))) / \sim$ in $S^{1} \times B^{3} \approx I \times\left(S^{3} \backslash N(p)\right) / \sim$. Let $N(\gamma)$ be a regular neighborhood of $\gamma$ in $W$ such that $S \cap N(\gamma)$ is an annulus, and choose an orientation-preserving diffeomorphism

$$
d:\left(S^{1} \times S^{2}, \partial A\right) \rightarrow(\partial N(\gamma), S \cap \partial N(\gamma)),
$$

the isotopy class of which is determined by a normal framing of $S$ along $\gamma$.

The concordance rim surgery $S(\gamma, C)$ of $S$ along $\gamma$ with pattern $C$ is obtained by gluing $\left(S^{1} \times B^{3}, A\right)$ to $(W \backslash N(\gamma), S \backslash N(\gamma))$ using the diffeomorphism $d$ (which we suppress in our notation). For an illustration, see Figure 2.1.

Remark 2.2. Alternatively, one could define concordance rim surgery as follows: Let $S$ be a properly embedded, smooth, oriented surface in the smooth 4 -manifold $W$, together with a simple closed curve $\gamma \subseteq S$. We denote by $T$ the rim torus around $\gamma$, which is the union of the fibers of the unit normal circle bundle of $S$ in $X$ over $\gamma$. Furthermore, let $\left(I \times S^{3}, C\right)$ be a self-concordance of a knot $K$ in $S^{3}$. Then we could define $S(\gamma, C)$ as the surface obtained by doing concordance surgery along the rim torus $T$ using the self-concordance $C$.

Kim [Kim06a, Lemma 2.4] showed the equivalence of the two definitions in the special case of twist rim surgery, which we review in the next section. We do not use this equivalence when proving our results, so we do not study the relationship between the two possible definitions of concordance rim surgery in general. 


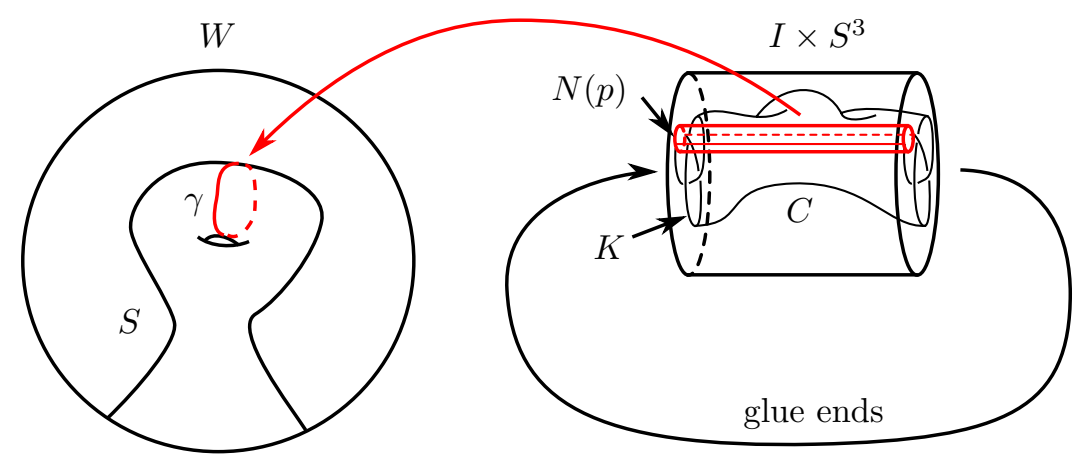

FIGURE 2.1. Concordance rim surgery on a surface $S$ along the curve $\gamma$ with pattern a self-concordance $C$ of a knot $K$.

2.2. Topological isotopy. In this section, we show that 1-twist rim surgery along a curve that bounds a topological disk in the surface complement preserves the topological isotopy type of the surface. We first review the definition of 1-twist rim surgery, due to Kim [Kim06a].

Definition 2.3. Let $K$ be an oriented knot in $S^{3}$, and let $T=\partial N(K)$ be the boundary of a regular neighborhood $N(K)$ of $K$. Fix an identification $T \approx S^{1} \times S^{1}$ such that $S^{1} \times\{\varphi\}$ is a meridian of $K$ for $\varphi \in S^{1}$. Let $V \approx T \times I$ be a regular neighborhood of $T$ in $S^{3} \backslash K$ such that $T \times\{0\} \subseteq N(K)$. Furthermore, choose a smooth monotonic function $f: \mathbb{R} \rightarrow I$ such that $f(t)=0$ for $t \leq 0$ and $f(t)=1$ for $t \geq 1$. We define an automorphism $\phi$ of $\left(S^{3}, K\right)$ by

$$
\phi(x)= \begin{cases}x & \text { if } x \notin V, \\ (\theta+2 \pi f(t), \varphi, t) & \text { if } x=(\theta, \varphi, t) \in V \approx S^{1} \times S^{1} \times I .\end{cases}
$$

For $n \in \mathbb{Z}$, the $n$-twist self-concordance of $K$ is given by the tuple

$$
\left(I \times S^{3}, I \times K, h_{0}, h_{1}\right),
$$

where $h_{i}:\{i\} \times S^{3} \rightarrow S^{3}$ are given by $h_{0}(0, x)=x$ and $h_{1}(1, x)=\phi^{n}(x)$. We denote the 1-twist self-concordance of $K$ by $\left(I \times S^{3}, K_{\mathrm{tw}}\right)$.

Let $S$ be a properly embedded, smooth, oriented surface in the compact 4-manifold $W$, and $\gamma \subseteq S$ a simple closed curve, together with a normal framing of $S$ along $\gamma$. Then we call $S\left(\gamma, K_{\mathrm{tw}}\right)$ the 1-twist rim surgery of $S$ along $\gamma$ with pattern $K$.

When $K$ is nontrivial, the knot cobordisms $\left(I \times S^{3}, K_{\text {tw }}\right)$ and $\left(I \times S^{3}, I \times K\right)$ are inequivalent, which follows from the work of Zeeman [Zee65].

Remark 2.4. The 1-twist self-concordance first appeared in the work of Zeeman [Zee65] in slightly different language. If $S^{2}$ is the standard 2-sphere in $S^{4}$ with equator $S^{1}$, and $K$ is a knot in $S^{3}$, then $S^{2}\left(S^{1}, K_{\text {tw }}\right)$ is the 1-twist-spun 2-knot obtained from $K$ in the terminology of Zeeman. Here, we use the normal framing of $S^{2}$ along $S^{1}$ induced by $D^{2}$.

In particular, Zeeman showed that, if $S$ is a 2 -sphere embedded in the 4-manifold $W$ that bounds a locally flat, topologically (resp. smoothly) embedded ball, and $K$ is a knot in $S^{3}$, then the 2-sphere $S\left(\gamma, K_{\mathrm{tw}}\right)$ also bounds a locally flat, topologically (resp. smoothly) embedded ball in $W$.

Theorem 2.5. Let $S$ be a properly embedded, oriented surface in a 4-manifold $W$. Suppose $\gamma \subseteq S$ a simple closed curve that bounds a locally flat, topologically embedded disk $D$ in $W$, such that int $(D) \cap$ $S=\emptyset$. Furthermore, let $C$ be a self-concordance of a knot in $S^{3}$ such that the concordance rim surgered 2-sphere $S^{2}\left(S^{1}, C\right)$ is topologically unknotted in $S^{4}$. Then $S(\gamma, C)$, the result of concordance rim surgery on $S$ along $\gamma$ with pattern $C$ and normal framing of $S$ along $\gamma$ given by $D$, is topologically isotopic to $S$.

Proof. We understand everything in this proof to take place in the topological, locally flat category. We use the existence and uniqueness of normal bundles up to ambient isotopy, and the existence of 
handle decompositions in this category, for which we refer the reader to Chapter 9 of the book of Freedman and Quinn [FQ90].

Let $D \times I$ be a thickening of $D$, such that $\partial D \times I \subseteq S$. Let $S^{\prime}$ be the surface obtained by smoothing the corners of $(S \backslash(\partial D \times I)) \cup(D \times \partial I)$. Choose a closed 4-ball $B$ containing $D \times I$ with $S^{\prime} \subseteq W \backslash \operatorname{int}(B)$ and $S^{\prime} \cap \partial B=D \times \partial I$; see the left and middle of Figure 2.2.

Note $S$ is obtained from $S^{\prime}$ by surgery along a 3-dimensional 1-handle with core arc $\eta=\{c\} \times I$, where $c$ is the center point of the disk $D$. The framing on $\gamma$ is determined by specifying that the result $S$ of this surgery is orientable.

Let $U$ be the (smoothed) symmetric difference of $S(\gamma, C)$ and $S^{\prime}$, and push $U$ off of $S^{\prime}$ and into $B$. By construction, the pair $(B, U)$ is homeomorphic to $\left(2 B^{4}, S^{2}\left(S^{1}, C\right)\right)$, where $2 B^{4} \subseteq \mathbb{R}^{4}$ is a ball of radius 2. By assumption, this implies that $U$ is an unknotted 2-sphere bounding a 3 -ball $\Delta$ in $B$. Now $S(\gamma, C)$ is obtained from $S^{\prime} \cup U$ by surgery along two 3-dimensional 1-handles with core arcs $\eta_{1}, \eta_{2} \subseteq B$, where $\eta_{1}=\{c\} \times[0,1 / 4]$ and $\eta_{2}=\{c\} \times[3 / 4,1]$. There are two choices of framings for $\eta_{1}$; the choice will determine the framing for $\eta_{2}$; see the right of Figure 2.2.
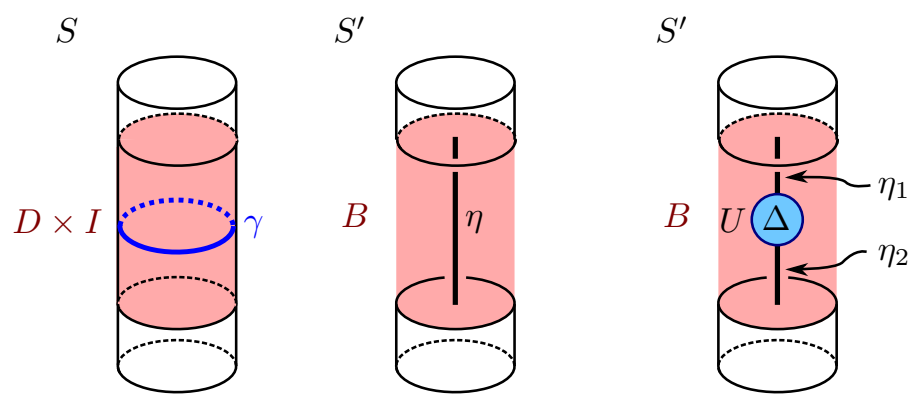

Figure 2.2. Left: A thickening of the locally flat disk $D$. The surface $S^{\prime}$ is obtained from $S$ by compressing along $D$. Middle: We obtain $S$ from $S^{\prime}$ by surgery along a 3-dimensional 1-handle with core $\eta$. Right: We obtain $S(\gamma, C)$ from $S^{\prime} \sqcup U$ by surgery along two 3 -dimensional 1 -handles with cores $\eta_{1}, \eta_{2}$. The sphere $U$ is the symmetric difference of $S(\gamma, C)$ and $S^{\prime}$, pushed off of $S^{\prime}$. Then $U$ is the result of concordance surgery on an unknotted sphere with pattern $C$, which is unknotted, by assumption.

Since $\pi_{1}(B \backslash \Delta) \cong 1$, we can isotope each $\eta_{i}$ rel boundary in $B$ to be disjoint from $\Delta$. By concatenating the 3-dimensional 1-handles about $\eta_{1}$ and $\eta_{2}$ with $\Delta$, we find that $S(\gamma, C)$ is obtained from $S^{\prime}$ by surgery along a 3-dimensional 1-handle with core arc $\eta^{\prime} \subseteq B$ (and with framing determined by the orientability of $S(\gamma, C)$ ). Since $\eta$ and $\eta^{\prime}$ are both contained in the ball $B$, we conclude that $\eta$ and $\eta^{\prime}$ are isotopic rel boundary as framed arcs in $W \backslash S^{\prime}$, and therefore that $S$ and $S(\gamma, C)$ are isotopic.

Remark 2.6. In Theorem 2.5, if we take $D$ to be smoothly embedded, then by the same argument in the smooth category we find that $S(\gamma, C)$ is smoothly isotopic to $S$.

When the disk $D$ is topologically but not smoothly embedded, we cannot conclude that $S(\gamma, C)$ is smoothly obtained from $S^{\prime}$ by surgery along a 3-dimensional 1-handle. Thus, the argument fails smoothly, and we do not necessarily expect that $S(\gamma, C)$ and $S$ are smoothly isotopic in general.

The most well-known situation in which concordance surgery on an unknotted sphere yields the unknot is the case of 1-twist rim surgery [Zee65].

Corollary 2.7. Let $S$ be a properly embedded, oriented surface in a 4-manifold $W$, and $\gamma \subseteq S$ a simple closed curve. Suppose $\gamma$ bounds a locally flat, topologically embedded disk $D$ in $W$, such that $\operatorname{int}(D) \cap S=\emptyset$. Then $S\left(\gamma, K_{\mathrm{tw}}\right)$ is topologically isotopic to $S$ for any knot $K$ in $S^{3}$, where the normal framing of $S$ along $\gamma$ is given by $D$. 
There are other concordance surgeries on the unknotted 2-sphere that yield the unknot; e.g., $n$ twist 1-roll rim surgery with pattern $K$, assuming $S_{n}^{3}(K)$ is a lens space; see Teragaito [Ter94] and Litherland [Lit79]. For our main examples, we will primarily consider 1-twist rim surgery.

Example 2.8. Let $S$ be a quasipositive Seifert surface of a knot $J$ in $S^{3}$, and $\gamma$ a homologically nontrivial simple closed curve on $S$ with trivial Alexander polynomial and trivial surface framing. Then $\gamma$ bounds a topological disk $D$ in $B^{4}$ that only intersects $S$ in $\gamma$. If we perform surgery along $D$, we obtain a topological surface $S^{\prime}$ bounding $J$ of smaller genus. Quasipositivity of $S$ implies it minimizes the slice genus. Hence, the topological slice genus of $J$ is less than its smooth slice genus, and the curve $\gamma$ does not bound a smooth disk in the complement of $S$. Compare Rudolph's counterexample to the locally flat Thom conjecture [Rud93, Section 5].

In particular, we can apply Theorem 2.5 to the pair $(S, \gamma)$ to see that the 1-twist rim surgery $S\left(\gamma, K_{\mathrm{tw}}\right)$ is topologically isotopic to $S$, for any knot $K$ in $S^{3}$. Since $\gamma$ does not bound a smooth disk in the complement of $S$, it is possible that $S$ and $S\left(\gamma, K_{\text {tw }}\right)$ are not smoothly isotopic. In the rest of the paper, we will prove that this is indeed the case whenever $\Delta_{K}(t) \neq 1$, using perturbed cobordism maps on link Floer homology.

\section{LiNK FlOER HOMOLOGY}

In this section, we review some background on the knot and link Floer homology groups of Ozsváth and Szabó [OS04] [OS08], as well as the constructions of link cobordism maps due to the first and third authors [Juh16] [Zem19b].

3.1. The link Floer homology groups. If $(\Sigma, \boldsymbol{\alpha}, \boldsymbol{\beta}, \mathbf{w}, \mathbf{z})$ is a Heegaard diagram for a multipointed link $(Y, \mathbb{L})$, then there are link Floer homology groups

$$
\widehat{H F L}(\Sigma, \boldsymbol{\alpha}, \boldsymbol{\beta}, \mathbf{w}, \mathbf{z}) \text { and } H F L^{-}(\Sigma, \boldsymbol{\alpha}, \boldsymbol{\beta}, \mathbf{w}, \mathbf{z}) \text {. }
$$

These are homologies of chain complexes $\widehat{C F L}(\Sigma, \boldsymbol{\alpha}, \boldsymbol{\beta}, \mathbf{w}, \mathbf{z})$ and $C F L^{-}(\Sigma, \boldsymbol{\alpha}, \boldsymbol{\beta}, \mathbf{w}, \mathbf{z})$, respectively, defined as follows.

Let $\mathbb{F}$ be the field of two elements, and $\mathbb{F}[v]$ the polynomial ring in the variable $v$. We consider the two half-dimensional tori

$$
\mathbb{T}_{\alpha}:=\alpha_{1} \times \cdots \times \alpha_{n} \quad \text { and } \quad \mathbb{T}_{\beta}:=\beta_{1} \times \cdots \times \beta_{n}
$$

in $\operatorname{Sym}^{n}(\Sigma)$, where $n=g(\Sigma)+|\mathbf{w}|-1$. Let $\widehat{C F L}(\Sigma, \boldsymbol{\alpha}, \boldsymbol{\beta}, \mathbf{w}, \mathbf{z})$ be the $\mathbb{F}$-vector space generated by intersection points $\mathbf{x} \in \mathbb{T}_{\alpha} \cap \mathbb{T}_{\beta}$. Furthermore, let $C F L^{-}(\Sigma, \boldsymbol{\alpha}, \boldsymbol{\beta}, \mathbf{w}, \mathbf{z})$ be the free $\mathbb{F}[v]$-module generated by intersection points $\mathbf{x} \in \mathbb{T}_{\alpha} \cap \mathbb{T}_{\beta}$.

The differential on $\widehat{C F L}(\Sigma, \boldsymbol{\alpha}, \boldsymbol{\beta}, \mathbf{w}, \mathbf{z})$ is given by the formula

$$
\partial \mathbf{x}=\sum_{\mathbf{y} \in \mathbb{T}_{\alpha} \cap \mathbb{T}_{\beta}} \sum_{\begin{array}{c}
\phi \in \pi_{2}(\mathbf{x}, \mathbf{y}) \\
\mu(\phi)=1 \\
( \\
n_{\mathbf{w}}(\phi)=n_{\mathbf{z}}(\phi)=0
\end{array}} \#(\mathcal{M}(\phi) / \mathbb{R}) \cdot \mathbf{y},
$$

where $\mathcal{M}(\phi)$ denotes the moduli space of pseudo-holomorphic disks in $\operatorname{Sym}^{n}(\Sigma)$ representing the class $\phi$. The differential on $C F L^{-}(\Sigma, \boldsymbol{\alpha}, \boldsymbol{\beta}, \mathbf{w}, \mathbf{z})$ is given by the formula

$$
\partial \mathbf{x}=\sum_{\mathbf{y} \in \mathbb{T}_{\alpha} \cap \mathbb{T}_{\beta}} \sum_{\substack{\phi \in \pi_{2}(\mathbf{x}, \mathbf{y}) \\ \mu(\phi)=1 \\ n_{\mathbf{w}}(\phi)=0}} \#(\mathcal{M}(\phi) / \mathbb{R}) v^{n_{\mathbf{z}}(\phi)} \cdot \mathbf{y},
$$

extended equivariantly over the action of $v$.

The chain complexes $\widehat{C F L}(\Sigma, \boldsymbol{\alpha}, \boldsymbol{\beta}, \mathbf{w}, \mathbf{z})$ and $C F L^{-}(\Sigma, \boldsymbol{\alpha}, \boldsymbol{\beta}, \mathbf{w}, \mathbf{z})$ have refinements over $\operatorname{Spin}^{c}$ structures. For $\mathfrak{s} \in \operatorname{Spin}^{c}(Y)$, we define $\widehat{C F L}(\Sigma, \boldsymbol{\alpha}, \boldsymbol{\beta}, \mathbf{w}, \mathbf{z}, \mathfrak{s})$ as the submodule generated by intersection points $\mathbf{x}$ which satisfy $\mathfrak{s}_{\mathbf{w}}(\mathbf{x})=\mathfrak{s}$, and similarly for $C F L^{-}$. When $c_{1}(\mathfrak{s})$ is torsion, and the link $\mathbb{L}$ is null-homologous, there are two Maslov gradings, which we denote by $\operatorname{gr}_{\mathbf{w}}$ and $\mathrm{gr}_{\mathbf{z}}$. With respect to $\left(\mathrm{gr}_{\mathbf{w}}, \mathrm{gr}_{\mathbf{z}}\right)$, the differential has bigrading $(-1,-1)$, and the action of $v$ has bigrading $(0,-2)$. 
3.2. Link cobordism maps. In this section, we summarize the functorial properties of link Floer homology, due to the first and third authors [Juh16] [Zem19b].

Definition 3.1. Suppose that $\left(Y_{0}, \mathbb{L}_{0}\right)$ and $\left(Y_{1}, \mathbb{L}_{1}\right)$ are two multi-pointed links. Write $\mathbb{L}_{i}=$ $\left(L_{i}, \mathbf{w}_{i}, \mathbf{z}_{i}\right)$ for $i \in\{0,1\}$. A decorated link cobordism from $\left(Y_{0}, \mathbb{L}_{0}\right)$ to $\left(Y_{1}, \mathbb{L}_{1}\right)$ consists of a pair $(W, \mathcal{S})$, satisfying the following:

(1) $W$ is a compact, oriented 4-manifold with $\partial W=-Y_{0} \cup Y_{1}$.

(2) $\mathcal{S}=(S, \mathcal{A})$, where $S$ is a compact, oriented, and properly embedded surface in $W$, such that $\partial S=-L_{0} \cup L_{1}$.

(3) $\mathcal{A}$ is a set of properly embedded $\operatorname{arcs}$ on $S$, whose endpoints are disjoint from $\mathbf{w}_{i}$ and $\mathbf{z}_{i}$ for $i \in\{0,1\}$, and such that each component of $L_{i} \backslash\left(\mathbf{w}_{i} \cup \mathbf{z}_{i}\right)$ contains exactly one endpoint of $\partial \mathcal{A}$. Furthermore, $S \backslash \mathcal{A}$ consists of two subsurfaces, denoted $\mathcal{S}_{\mathbf{w}}$ and $\mathcal{S}_{\mathbf{z}}$, that meet along $\mathcal{A}$, such that $\mathbf{w}_{i} \subseteq \mathcal{S}_{\mathbf{w}}$ and $\mathbf{z}_{i} \subseteq \mathcal{S}_{\mathbf{z}}$.

(4) Each component of $W$ contains a component of $S$, and each component of $S$ contains a component of $\mathcal{A}$.

For a decorated link cobordism $(W, \mathcal{S}):\left(Y_{0}, \mathbb{L}_{0}\right) \rightarrow\left(Y_{1}, \mathbb{L}_{1}\right)$, the first author [Juh16] constructed a cobordism map

$$
\hat{F}_{W, \mathcal{S}}: \widehat{H F L}\left(Y_{0}, \mathbb{L}_{0}\right) \rightarrow \widehat{H F L}\left(Y_{1}, \mathbb{L}_{1}\right) .
$$

The third author [Zem19b] subsequently constructed $\mathbb{F}[v]$-equivariant maps

$$
F_{W, \mathcal{S}}^{-}: \operatorname{HFL}^{-}\left(Y_{0}, \mathbb{L}_{0}\right) \rightarrow H F L^{-}\left(Y_{1}, \mathbb{L}_{1}\right)
$$

The construction in [Zem19b] also induces a cobordism map on $\widehat{H F L}$, which is of a different flavor than the one in [Juh16]. The first and third authors [JZ18b, Theorem 1.4] proved that the two constructions give the same maps on $\widehat{H F L}$.

3.3. Duality and link Floer homology. We now recall some basic results about duality and link Floer homology, which feature prominently in the functorial properties of the transverse invariants. Firstly, if $\mathcal{H}=(\Sigma, \boldsymbol{\alpha}, \boldsymbol{\beta}, \mathbf{w}, \mathbf{z})$ is a Heegaard link diagram for $(Y, \mathbb{L})$, then $\mathcal{H}^{\vee}=(\Sigma, \boldsymbol{\beta}, \boldsymbol{\alpha}, \mathbf{w}, \mathbf{z})$ is a diagram for $(-Y,-\mathbb{L})$, where $-\mathbb{L}=(-L, \mathbf{w}, \mathbf{z})$ denotes $\mathbb{L}$, with orientation reversed.

There is a canonical isomorphism

$$
\widehat{C F L}(\Sigma, \boldsymbol{\beta}, \boldsymbol{\alpha}, \mathbf{w}, \mathbf{z}) \cong(\widehat{C F L}(\Sigma, \boldsymbol{\alpha}, \boldsymbol{\beta}, \mathbf{w}, \mathbf{z}))^{\vee},
$$

where $\vee$ denotes duality of $\mathbb{F}$-vector spaces; see [OS04, Proposition 3.7]. Similarly, the chain complexes $C F L^{-}(\Sigma, \boldsymbol{\beta}, \boldsymbol{\alpha}, \mathbf{w}, \mathbf{z})$ and $C F L^{-}(\Sigma, \boldsymbol{\alpha}, \boldsymbol{\beta}, \mathbf{w}, \mathbf{z})^{\vee}$ are canonically isomorphic, where $\vee$ now denotes the dual as a chain complex over $\mathbb{F}[v]$.

The link cobordism maps also satisfy an analogous duality property. If $(W, \mathcal{S}):\left(Y_{0}, \mathbb{L}_{0}\right) \rightarrow\left(Y_{1}, \mathbb{L}_{1}\right)$ is a decorated link cobordism, then turning around $(W, \mathcal{S})$ gives a link cobordism

$$
(W, \mathcal{S})^{\vee}:=\left(W^{\vee}, \mathcal{S}^{\vee}\right):\left(-Y_{1},-\mathbb{L}_{1}\right) \rightarrow\left(-Y_{0},-\mathbb{L}_{0}\right) .
$$

Furthermore, using the description of the link cobordism maps in terms of elementary cobordisms from [Zem19b], it is straightforward to adapt [OS06, Theorem 3.5] to obtain that

$$
F_{(W, \mathcal{S})^{\vee}}^{\circ}=\left(F_{W, \mathcal{S}}^{\circ}\right)^{\vee}
$$

for $\circ \in\{\wedge,-\}$.

\section{Perturbed sutured Floer homology}

Sutured Floer homology is an invariant of sutured manifolds, due to the first author [Juh06]. Perturbed sutured Floer homology, introduced by the first and third authors [JZ18a], is a refinement for sutured manifolds equipped with a collection of closed 2-forms.

Sutured Floer homology perturbed by $n$ closed 2 -forms has coefficients in the group ring $\mathbb{F}\left[\mathbb{R}^{n}\right]$, which we note contains $\mathbb{F}\left[\mathbb{N}^{n}\right]$ and $\mathbb{F}\left[\mathbb{Z}^{n}\right]$. These are the rings of polynomials and Laurent polynomials, 
respectively. If $\left(a_{1}, \ldots, a_{n}\right) \in \mathbb{R}^{n}$, we write $e^{\left(a_{1}, \ldots, a_{n}\right)}$ for the corresponding element of the group ring, which we think of as the monomial $z_{1}^{a_{1}} \cdots z_{n}^{a_{n}}$.

If $\boldsymbol{\omega}=\left(\omega_{1}, \ldots, \omega_{n}\right)$ is a tuple of closed 2 -forms on a manifold $X$, then there is an action of the group $C_{2}(X ; \mathbb{Z})$ of smooth 2 -chains on $\mathbb{F}\left[\mathbb{R}^{n}\right]$, given by

$$
e^{h} \cdot e^{\left(a_{1}, \ldots, a_{n}\right)}=e^{\left(a_{1}+\int_{h} \omega_{1}, \ldots, a_{n}+\int_{h} \omega_{n}\right)} .
$$

We write $\mathbb{F}\left[\mathbb{R}^{n}\right]_{\boldsymbol{\omega}}$ for $\mathbb{F}\left[\mathbb{R}^{n}\right]$ equipped with this action.

Definition 4.1. If $x \in \mathbb{F}\left[\mathbb{R}^{n}\right]$, then we say that $x$ is projectively integral if

$$
x=m \cdot y
$$

for some $y \in \mathbb{F}\left[\mathbb{Z}^{n}\right]$ and monomial $m=e^{\left(a_{1}, \ldots, a_{n}\right)} \in \mathbb{F}\left[\mathbb{R}^{n}\right]$. More generally, if $M_{0}$ is an $\mathbb{F}$-vector space, and

$$
M:=M_{0} \otimes \mathbb{F}\left[\mathbb{R}^{n}\right]
$$

we say that $x \in M$ is projectively integral if there is some monomial $m \in \mathbb{F}\left[\mathbb{R}^{n}\right]$ such that $m \cdot x \in$ $M_{0} \otimes \mathbb{F}\left[\mathbb{Z}^{n}\right]$.

Definition 4.2. If $X$ is a smooth manifold, we say that a closed 2-form $\omega \in \Omega^{2}(X)$ is integral if $\int_{F} \omega \in \mathbb{Z}$ for any closed, singular 2-chain $F \in C_{2}(X ; \mathbb{Z})$.

If $\boldsymbol{\omega}$ is an $n$-tuple of closed 2 -forms on a sutured manifold $(M, \gamma)$, the first and third authors [JZ18a] described a perturbed version of sutured Floer homology, denoted $S F H\left(M, \gamma ; \mathbb{F}\left[\mathbb{R}^{n}\right]_{\boldsymbol{\omega}}\right)$. If $\mathcal{W}$ is a cobordism between the sutured manifolds $\left(M_{0}, \gamma_{0}\right)$ and $\left(M_{1}, \gamma_{1}\right)$, and $\boldsymbol{\omega}$ is a collection of closed 2 -forms on $\mathcal{W}$, then the first and third authors also constructed a perturbed version of the cobordism map

$$
F_{\mathcal{W} ; \boldsymbol{\omega}}: \operatorname{SFH}\left(M_{0}, \gamma_{0} ; \mathbb{F}\left[\mathbb{R}^{n}\right]_{\boldsymbol{\omega}_{0}}\right) \rightarrow \operatorname{SFH}\left(M_{1}, \gamma_{1} ; \mathbb{F}\left[\mathbb{R}^{n}\right]_{\boldsymbol{\omega}_{1}}\right),
$$

where $\boldsymbol{\omega}_{i}=\left.\boldsymbol{\omega}\right|_{M_{i}}$. A sketch of the construction may be found later in this section.

The main technical result of this section is the following:

Proposition 4.3. Suppose that $S \subseteq B^{4}$ is a properly embedded, oriented surface intersecting $S^{3}$ in a knot $K$, and $\boldsymbol{\omega}=\left(\omega_{1}, \ldots, \omega_{n}\right)$ is a collection of closed, integral 2-forms on $B^{4} \backslash N(S)$ that vanish on $S^{3} \backslash N(K)$. Then, for any dividing set $\mathcal{A}$ on $S$, the element

$$
F_{B^{4}, \mathcal{S} ; \boldsymbol{\omega}}(1) \in \widehat{H F K}\left(S^{3}, K\right) \otimes \mathbb{F}\left[\mathbb{R}^{n}\right]
$$

is projectively integral, where $\mathcal{S}=(S, \mathcal{A})$ and $F_{B^{4}, \mathcal{S} ; \omega}$ is the cobordism map on perturbed sutured Floer homology [JZ18a] over $\mathbb{F}\left[\mathbb{R}^{n}\right]$ induced by the sutured manifold cobordism complementary to $\mathcal{S}$ from the empty sutured manifold to $S^{3}(K)$, where $\operatorname{SFH}\left(S^{3}(K)\right) \cong \widehat{H F K}\left(S^{3}, K\right)$.

4.1. A totally twisted version of sutured Floer homology. To show Proposition 4.3, it is convenient to lift the map on perturbed sutured Floer homology to a totally twisted map, in the spirit of the version described by Ozsváth and Szabó for ordinary Heegaard Floer homology [OS06, Section 2.7].

If $X$ is a topological space, write $C_{k}(X)$ for the group of smooth, integral, singular $k$-chains in $X$. Let $B_{2}(X)$ be the image of $\partial: C_{3}(X) \rightarrow C_{2}(X)$, and write $\left(C_{2} / B_{2}\right)(X)$ for the quotient $C_{2}(X) / B_{2}(X)$.

Let $(M, \gamma)$ be a balanced sutured manifold with admissible diagram $(\Sigma, \boldsymbol{\alpha}, \boldsymbol{\beta})$, and let $\mathfrak{s}$ be a relative $\operatorname{Spin}^{c}$ structure on $(M, \gamma)$. If $L$ is a $\mathbb{F}\left[\left(C_{2} / B_{2}\right)(M)\right]$-module, we define the twisted sutured Floer complex

$$
\underline{C F}(\Sigma, \boldsymbol{\alpha}, \boldsymbol{\beta}, \underline{\mathfrak{s}} ; L)
$$

to be the group generated by elements $\mathbf{x} \otimes h$, where $\mathbf{x} \in \mathbb{T}_{\alpha} \cap \mathbb{T}_{\beta}$ satisfies $\mathfrak{s}(\mathbf{x})=\mathfrak{s}$, and $h \in L$. The differential is given by

$$
\partial(\mathbf{x} \otimes h)=\sum_{\mathbf{y} \in \mathbb{T}_{\alpha} \cap \mathbb{T}_{\beta}} \sum_{\substack{\phi \in \pi_{2}(\mathbf{x}, \mathbf{y}) \\ \mu(\phi)=1}} \#(\mathcal{M}(\phi) / \mathbb{R}) \cdot \mathbf{y} \otimes h \cdot e^{\widetilde{\mathcal{D}}(\phi)},
$$


where $\widetilde{\mathcal{D}}(\phi) \in C_{2}(M)$ is obtained by coning off the domain of $\phi$ using sets of compressing disks for $\boldsymbol{\alpha}$ and $\boldsymbol{\beta}$, which are implicit in the construction, as in [JZ18a, Section 2.2]. If $\omega$ is an $n$-tuple of closed 2-form on $M$, then the perturbed complex $C F\left(\Sigma, \boldsymbol{\alpha}, \boldsymbol{\beta}, \underline{\mathfrak{s}} ; \mathbb{F}[\mathbb{R}]_{\boldsymbol{\omega}}\right)$ is the tensor product

$$
\underline{C F}\left(\Sigma, \boldsymbol{\alpha}, \boldsymbol{\beta}, \underline{\mathfrak{s}} ; \mathbb{F}\left[\left(C_{2} / B_{2}\right)(M)\right]\right) \otimes_{\mathbb{F}\left[\left(C_{2} / B_{2}\right)(M)\right]} \mathbb{F}\left[\mathbb{R}^{n}\right]_{\boldsymbol{\omega}}
$$

where $\mathbb{F}\left[\left(C_{2} / B_{2}\right)(M)\right]$ acts on $\mathbb{F}\left[\mathbb{R}^{n}\right]$ as in equation $(3)$.

We write

$$
\underline{C F}(\Sigma, \boldsymbol{\alpha}, \boldsymbol{\beta} ; L)=\bigoplus_{\underline{\mathfrak{s}} \in \operatorname{Spin}^{c}(M, \gamma)} \underline{C F}(\Sigma, \boldsymbol{\alpha}, \boldsymbol{\beta}, \underline{\mathfrak{s}} ; L)
$$

There are inclusion and projection maps

$$
i_{\underline{\mathfrak{s}}}: \underline{C F}(\mathcal{H}, \underline{\mathfrak{s}} ; L) \rightarrow \underline{C F}(\mathcal{H} ; L) \text { and } \pi_{\underline{\mathfrak{s}}}: \underline{C F}(\mathcal{H} ; L) \rightarrow \underline{C F}(\mathcal{H}, \underline{\mathfrak{s}} ; L),
$$

where $\mathcal{H}=(\Sigma, \boldsymbol{\alpha}, \boldsymbol{\beta})$.

The chain complexes $\underline{C F}(\Sigma, \boldsymbol{\alpha}, \boldsymbol{\beta}, \underline{\mathfrak{s}} ; L)$ for a given sutured manifold $(M, \gamma)$ and $\underline{\mathfrak{s}} \in \operatorname{Spin}^{c}(M, \gamma)$ form a projective transitive system that we denote by $\underline{C F}(M, \gamma, \underline{\mathfrak{s}} ; L)$. I.e., the only monodromy of the transition maps is multiplication by $e^{h}$ for $h \in\left(C_{2} / B_{2}\right)(M)$, up to chain homotopy; see [JZ18a, Section 2.1]. The transition maps for changing diagrams are defined using straightforward extensions of the formulas below for cobordism maps; also see [JZ18a, Section 6]. In general, summing over $\operatorname{Spin}^{c}$ structures will not produce a natural invariant, as in [JZ18a, Section 6.5].

Let $\mathcal{W}=(W, Z,[\xi])$ be a balanced sutured manifold cobordism from $\left(M_{0}, \gamma_{0}\right)$ to $\left(M_{1}, \gamma_{1}\right)$. We can view $\mathbb{F}\left[\left(C_{2} / B_{2}\right)(W)\right]$ as a $\mathbb{F}\left[\left(C_{2} / B_{2}\right)\left(M_{k}\right)\right]$-module using the embedding $i_{k}: M_{k} \hookrightarrow W$ for $k \in\{0,1\}$. We now describe a totally twisted map

$$
\underline{F}_{\mathcal{W}}: \underline{C F}\left(M_{0}, \gamma_{0} ; \mathbb{F}\left[\left(C_{2} / B_{2}\right)\left(M_{0}\right)\right]\right) \rightarrow \underline{C F}\left(M_{1}, \gamma_{1} ; \mathbb{F}\left[\left(C_{2} / B_{2}\right)(W)\right]\right),
$$

as follows. We decompose $\mathcal{W}$ into a boundary cobordism $\mathcal{W}^{\partial}$ and a special cobordism $\mathcal{W}^{s}$, as in [Juh16]. We further decompose $\mathcal{W}^{s}$ into cylinders, and 1-handle, 2-handle, and 3-handle cobordisms. As in [JZ18a, Proposition 2.9], only the map

$$
\pi_{\underline{\mathfrak{s}}_{1}} \circ \underline{F}_{\mathcal{W}} \circ i_{\underline{\mathfrak{s}}_{0}}: \underline{C F}\left(M_{0}, \gamma_{0}, \underline{\mathfrak{s}}_{0} ; \mathbb{F}\left[\left(C_{2} / B_{2}\right)\left(M_{0}\right)\right]\right) \rightarrow \underline{C F}\left(M_{1}, \gamma_{1}, \underline{\mathfrak{s}}_{1} ; \mathbb{F}\left[\left(C_{2} / B_{2}\right)(W)\right]\right)
$$

is well-defined, up to multiplication by $e^{h}$ for $h \in\left(C_{2} / B_{2}\right)(W)$, and chain homotopy.

We begin by defining the totally twisted map for cylinders. If $W$ has a Morse function $f$ with no critical points and gradient-like vector field $v$, then the flow of $v / v(f)$ gives a diffeomorphism between $W$ and $I \times M_{0}$. If $\mathcal{H}_{0}$ is an admissible diagram for $M_{0}$, we obtain an admissible diagram $\mathcal{H}_{1}$ for $\left(M_{1}, \gamma_{1}\right)$ by using the flow of $v / v(f)$. If $\mathbf{x}$ is an intersection point for $\mathcal{H}_{0}$, there is a corresponding intersection point of $\mathcal{H}_{1}$, for which we write $v_{*}(\mathbf{x})$. We let $\Gamma_{\mathbf{x}}$ be the singular 2-chain obtained by sweeping out $\gamma_{\mathbf{x}}$ under the flow of $v / v(f)$. The twisted map for $\mathcal{W}$ in this case is

$$
\underline{F}_{\mathcal{W}}\left(\mathbf{x} \otimes e^{h}\right)=v_{*}(\mathbf{x}) \otimes e^{\left(i_{0}\right)_{*}(h)+\Gamma_{\mathbf{x}}},
$$

where $h \in\left(C_{2} / B_{2}\right)\left(M_{0}\right)$, and $\left(i_{0}\right)_{*}:\left(C_{2} / B_{2}\right)\left(M_{0}\right) \rightarrow\left(C_{2} / B_{2}\right)(W)$ is induced by the embedding $i_{0}: M_{0} \hookrightarrow W$. The twisted cobordism maps for 1-handles and 3-handles are defined using a similar formula, so we leave the details to the reader. Compare [JZ18a, Section 7.3].

We now focus on 2-handle cobordisms, and follow [JZ18a, Section 7.4]. We pick a Morse function $f$ on $W$ with gradient-like vector field $v$ that is Morse-Smale and has only index 2 critical points. Let $\left(\Sigma, \boldsymbol{\alpha}, \boldsymbol{\beta}, \boldsymbol{\beta}^{\prime}\right)$ be a Heegaard triple subordinate to a bouquet for the framed link in $M_{0}$ induced by $(f, v)$. We obtain an embedding of $W_{\alpha, \beta, \beta^{\prime}}$ into $W$, which is well-defined up to isotopy. If $\psi \in \pi_{2}\left(\mathbf{x}, \Theta_{\beta, \beta^{\prime}}, \mathbf{y}\right)$ is a class of triangles with $\mathbf{x} \in \mathbb{T}_{\alpha} \cap \mathbb{T}_{\beta}$ and $\mathbf{y} \in \mathbb{T}_{\alpha} \cap \mathbb{T}_{\beta^{\prime}}$, we obtain a 2-chain $\widetilde{\mathcal{D}}(\psi)$ in $W$ by coning off the domain $\mathcal{D}(\psi)$. The 2-handle map is defined via the formula

$$
\underline{F}_{\mathcal{W}}\left(\mathbf{x} \otimes e^{h}\right)=\sum_{\substack{\mathbf{y} \in \mathbb{T}_{\alpha} \cap \mathbb{T}_{\beta^{\prime}} \\ \mathfrak{s}(\mathbf{y})=\underline{\mathfrak{s}}_{1}}} \sum_{\substack{\psi \in \pi_{2}\left(\mathbf{x}, \Theta_{\beta, \beta^{\prime}}, \mathbf{y}\right) \\ \mu(\psi)=0}} \# \mathcal{M}(\psi) \cdot \mathbf{y} \otimes e^{\left(i_{0}\right)_{*}(h)+\widetilde{\mathcal{D}}(\psi)},
$$

where $\mathbf{x} \in \mathbb{T}_{\alpha} \cap \mathbb{T}_{\beta}$ satisfies $\mathfrak{s}(\mathbf{x})=\underline{\mathfrak{s}}_{0}$, and $h \in\left(C_{2} / B_{2}\right)\left(M_{0}\right)$.

The contact gluing map extends to this setting, as follows. Let $(M, \gamma)$ be a sutured submanifold of $\left(M^{\prime}, \gamma^{\prime}\right)$, and $\xi$ a positive contact structure on $M^{\prime} \backslash \operatorname{int}(M)$ that induces the dividing set $\gamma \cup \gamma^{\prime}$. 
For $\underline{\mathfrak{s}} \in \operatorname{Spin}^{c}(M, \gamma)$ represented by the nowhere vanishing vector field $v$ on $M$, we obtain $\underline{\mathfrak{s}}^{\prime} \in$ $\operatorname{Spin}^{c}\left(M^{\prime}, \gamma^{\prime}\right)$ by gluing $v$ to $\xi^{\perp}$. Then the contact gluing map

$$
\underline{\Phi}_{\xi}: \underline{C F}\left(-M, \gamma, \underline{\mathfrak{s}} ; \mathbb{F}\left[\left(C_{2} / B_{2}\right)(M)\right]\right) \rightarrow \underline{C F}\left(-M^{\prime}, \gamma^{\prime}, \underline{\mathfrak{s}^{\prime}} ; \mathbb{F}\left[\left(C_{2} / B_{2}\right)\left(M^{\prime}\right)\right]\right)
$$

is defined via the formula

$$
\underline{\Phi}_{\xi}\left(\mathbf{x} \otimes e^{h}\right)=\Phi_{\xi}(\mathbf{x}) \otimes e^{i_{*}(h)},
$$

where $\mathfrak{s}(\mathbf{x})=\underline{\mathfrak{s}}^{\prime}$, the map $i_{*}:\left(C_{2} / B_{2}\right)(M) \rightarrow\left(C_{2} / B_{2}\right)\left(M^{\prime}\right)$ is induced by the inclusion of $M$ into $M^{\prime}$, and $\Phi_{\xi}$ is the untwisted gluing map.

Finally, as in [JZ18a, Section 7.5], we define the totally twisted cobordism map $\underline{F}_{\mathcal{W}}$ for a general balanced cobordism $\mathcal{W}$ by composing the totally twisted contact gluing map to obtain $\underline{F}_{\mathcal{W}^{2}}$, followed by the totally twisted maps for the cylinders and handle cobordisms to obtain $\underline{F}_{\mathcal{W} s}$.

One may follow the proof of invariance of the perturbed cobordism maps from [JZ18a, Section 7.5] to see that the $\operatorname{Spin}^{c}$ restricted totally twisted cobordism map $\pi_{\underline{\mathfrak{s}}_{1}} \circ \underline{F}_{\mathcal{W}} \circ i_{\underline{\mathfrak{s}}_{0}}$ is well-defined, up to overall multiplication by $e^{h}$ for $h \in\left(C_{2} / B_{2}\right)(W)$, and chain homotopy.

By construction, if $\boldsymbol{\omega}=\left(\omega_{1}, \ldots, \omega_{n}\right)$ is a collection of closed 2-forms on $W$, then one obtains the perturbed map $F_{\mathcal{W} ; \boldsymbol{\omega}}$ by tensoring with the group ring $\mathbb{F}\left[\mathbb{R}^{n}\right]_{\boldsymbol{\omega}}$, which is a module over $\left(C_{2} / B_{2}\right)(W)$ with the action shown in equation (3); i.e.,

$$
F_{\mathcal{W} ; \omega}=\underline{F}_{\mathcal{W}} \otimes 1_{\mathbb{F}\left[\mathbb{R}^{n}\right]_{\omega}} .
$$

4.2. Proof of Proposition 4.3. We now proceed with the main details of the proof of Proposition 4.3. We begin with a lemma:

Lemma 4.4. Suppose that $\mathcal{W}=(W, Z,[\xi]):\left(M_{0}, \gamma_{0}\right) \rightarrow\left(M_{1}, \gamma_{1}\right)$ is a balanced sutured manifold cobordism, and $\mathcal{H}_{0}$ and $\mathcal{H}_{1}$ are admissible diagrams for $\left(M_{0}, \gamma_{0}\right)$ and $\left(M_{1}, \gamma_{1}\right)$, respectively. Suppose $\mathbf{x}_{0}$ and $\mathbf{x}_{0}^{\prime}$ are intersection points on $\mathcal{H}_{0}$, and $\mathbf{x}_{1}$ and $\mathbf{x}_{1}^{\prime}$ are intersection points on $\mathcal{H}_{1}$, such that

$$
\mathfrak{s}\left(\mathbf{x}_{0}\right)=\mathfrak{s}\left(\mathbf{x}_{0}^{\prime}\right) \in \operatorname{Spin}^{c}\left(M_{0}, \gamma_{0}\right) \quad \text { and } \quad \mathfrak{s}\left(\mathbf{x}_{1}\right)=\mathfrak{s}\left(\mathbf{x}_{1}^{\prime}\right) \in \operatorname{Spin}^{c}\left(M_{1}, \gamma_{1}\right) .
$$

Suppose $\mathbf{x}_{1} \otimes e^{h_{\mathbf{x}_{0}}}$ appears as a summand of $\underline{F}_{\mathcal{W}}\left(\mathbf{x}_{0}\right)$ and $\mathbf{x}_{1}^{\prime} \otimes e^{h_{\mathbf{x}_{0}^{\prime}}}$ appears as a summand of $\underline{F}_{\mathcal{W}}\left(\mathbf{x}_{0}^{\prime}\right)$, for some $h_{\mathbf{x}_{0}}, h_{\mathbf{x}_{0}^{\prime}} \in\left(C_{2} / B_{2}\right)(W)$. Let $\phi_{0} \in \pi_{2}\left(\mathbf{x}_{0}, \mathbf{x}_{0}^{\prime}\right)$ and $\phi_{1} \in \pi_{2}\left(\mathbf{x}_{1}, \mathbf{x}_{1}^{\prime}\right)$. Then

$$
h_{\mathbf{x}_{0}}-h_{\mathbf{x}_{0}^{\prime}}+\left(i_{1}\right)_{*} \widetilde{\mathcal{D}}\left(\phi_{1}\right)-\left(i_{0}\right)_{*} \widetilde{\mathcal{D}}\left(\phi_{0}\right)
$$

is a closed 2-chain in $W$, where $\left(i_{0}\right)_{*}$ and $\left(i_{1}\right)_{*}$ are the maps induced by the inclusions of $M_{0}$ and $M_{1}$ into $W$.

Proof. It is sufficient to show the claim separately for the twisted contact gluing map, the 1-handle, 2-handle, and 3-handle maps, as well as the transition maps for changing the Heegaard diagram.

The claim for the contact gluing maps, the 1-handle maps, and the 3-handle maps are straightforward, so we focus on the 2-handle maps. The claim for the transition maps for changing the Heegaard diagram is an easy modification of the argument we present for 2-handles. Suppose $(f, v)$ is a MorseSmale pair on $W$, and $f$ has only index 2 critical points. Suppose $\left(\Sigma, \boldsymbol{\alpha}, \boldsymbol{\beta}, \boldsymbol{\beta}^{\prime}\right)$ is a Heegaard triple subordinate to a bouquet for the framed link in $M_{0}$ induced by $(f, v)$. We obtain an embedding of the 3 -ended cobordism $W_{\alpha, \beta, \beta^{\prime}}$ into $W$. Suppose $\psi \in \pi_{2}\left(\mathbf{x}_{0}, \Theta_{\beta, \beta^{\prime}}, \mathbf{x}_{1}\right)$ and $\psi^{\prime} \in \pi_{2}\left(\mathbf{x}_{0}^{\prime}, \Theta_{\beta, \beta^{\prime}}, \mathbf{x}_{1}^{\prime}\right)$. Let $\phi_{0} \in \pi_{2}\left(\mathbf{x}_{0}, \mathbf{x}_{0}^{\prime}\right)$ and $\phi_{1} \in \pi_{2}\left(\mathbf{x}_{1}, \mathbf{x}_{1}^{\prime}\right)$ be classes of disks, as in the statement.

We simply note that

$$
\begin{gathered}
\partial \widetilde{\mathcal{D}}\left(\phi_{0}\right)=\gamma_{\mathbf{x}_{0}^{\prime}}-\gamma_{\mathbf{x}_{0}}, \quad \partial \widetilde{\mathcal{D}}\left(\phi_{1}\right)=\gamma_{\mathbf{x}_{1}^{\prime}}-\gamma_{\mathbf{x}_{1}}, \\
\partial \widetilde{\mathcal{D}}(\psi)=C_{\alpha, \beta, \beta^{\prime}}+\gamma_{\mathbf{x}_{1}}-\gamma_{\Theta_{\beta, \beta^{\prime}}}-\gamma_{\mathbf{x}_{0}}, \quad \text { and } \quad \partial \widetilde{\mathcal{D}}\left(\psi^{\prime}\right)=C_{\alpha, \beta, \beta^{\prime}}+\gamma_{\mathbf{x}_{1}^{\prime}}-\gamma_{\Theta_{\beta, \beta^{\prime}}}-\gamma_{\mathbf{x}_{0}^{\prime}},
\end{gathered}
$$

where $C_{\alpha, \beta, \beta^{\prime}}$ is the 1 -chain in $W$, defined as follows. The 4-manifold $W_{\alpha, \beta, \beta^{\prime}}$ is constructed by gluing $\Sigma \times \Delta, U_{\alpha} \times e_{a}, U_{\beta} \times e_{\beta}$, and $U_{\beta^{\prime}} \times e_{\beta^{\prime}}$, where $\Delta$ denotes a triangle, $U_{\alpha}, U_{\beta}$, and $U_{\beta^{\prime}}$ are standard sutured compression bodies, and $e_{\alpha}, e_{\beta}$, and $e_{\beta^{\prime}}$ are intervals that are identified with the sides of the triangle $\Delta$. Given a collection of compressing disks for $U_{\alpha}$, we write $c_{\alpha}$ for their center points, and we define the 1-chain $C_{\alpha}=e_{\alpha} \times c_{\alpha}$. We define the 1-chains $C_{\beta}$ and $C_{\beta^{\prime}}$ similarly. The 
1-chain $C_{\alpha, \beta, \beta^{\prime}}$ is the sum of $C_{\alpha}, C_{\beta}$, and $C_{\beta^{\prime}}$ (in particular, it is independent of the choice of triangle or intersection point). By equation (5), we have

$$
\partial \widetilde{\mathcal{D}}(\psi)-\partial \widetilde{\mathcal{D}}\left(\psi^{\prime}\right)+\partial\left(i_{1}\right)_{*} \widetilde{\mathcal{D}}\left(\phi_{1}\right)-\partial\left(i_{0}\right)_{*} \widetilde{\mathcal{D}}\left(\phi_{0}\right)=0,
$$

and the result follows.

Proof of Proposition 4.3. Write $\mathcal{W}$ for the sutured manifold cobordism complementary to $S$, viewed as a cobordism from from $\emptyset$ to $S^{3}(K)$, the sutured manifold complementary to $K$.

The map $F_{\mathcal{W} ; \boldsymbol{\omega}}$ satisfies an Alexander grading formula; see [JM17, p. 3] and [Zem19a, Theorem 1.4]. In particular, all summands of $F_{\mathcal{W} ; \boldsymbol{\omega}}(1)$ reside in the same Alexander grading, which is equivalent to representing the same relative $\operatorname{Spin}^{c}$ structure on $S^{3}(K)$.

We apply Lemma 4.4 to see that, if $\mathbf{x} \otimes e^{h}$ and $\mathbf{x}^{\prime} \otimes e^{h^{\prime}}$ are summands of the totally twisted map $\underline{F}_{\mathcal{W}}(1)$ for some $h, h^{\prime} \in\left(C_{2} / B_{2}\right)(W)$, and $\phi \in \pi_{2}\left(\mathbf{x}, \mathbf{x}^{\prime}\right)$ is a class of disks, then

$$
h-h^{\prime}+\widetilde{\mathcal{D}}(\phi)
$$

is a closed 2-chain. We obtain that

$$
\int_{h-h^{\prime}+\widetilde{\mathcal{D}}(\phi)} \omega_{i} \in \mathbb{Z}
$$

for every $i \in\{1, \ldots, n\}$, since $\omega_{i}$ is integral by assumption. Note that $\omega_{i}$ also vanishes on $S^{3} \backslash N(K)$ by assumption, so $\int_{\widetilde{\mathcal{D}}(\phi)} \omega_{i}=0$, and hence

$$
\int_{h} \omega_{i}-\int_{h^{\prime}} \omega_{i} \in \mathbb{Z}
$$

Since the perturbed map is obtained from the totally twisted one by tensoring with $\mathbb{F}\left[\mathbb{R}^{n}\right]_{\boldsymbol{\omega}}$, we conclude that, after multiplying $F_{\mathcal{W} ; \boldsymbol{\omega}}(1)$ by some $e^{\left(a_{1}, \ldots, a_{n}\right)}$, we obtain an element of $\widehat{H F K}(K) \otimes$ $\mathbb{F}\left[\mathbb{Z}^{n}\right]$, concluding the proof.

\section{Concordance Rim Surgery and Knot Floer homology}

In this section, we compute the effect of concordance rim surgery on the perturbed cobordism maps. Suppose $S$ is a properly embedded, oriented surface in $B^{4}$ intersecting $S^{3}$ in a knot $K$. We identify a neighborhood $N(S)$ of $S$ with $S \times D^{2}$. It is straightforward to investigate the MayerVietoris sequence for the decomposition of $B^{4}$ as the union of $B^{4} \backslash N(S)$ and $N(S)$ to see that

$$
H^{2}\left(B^{4} \backslash N(S), S^{3} \backslash N(K)\right) \cong H^{1}(S, \partial S) .
$$

Geometrically, this isomorphism can be described by taking a properly embedded surface in $B^{4} \backslash N(S)$ with boundary in $\partial N(S)$, taking its intersection with $S \times S^{1}$, and projecting to $S$. Hence, we can identify a basis of $H^{2}\left(B^{4} \backslash N(S), S^{3} \backslash N(K)\right)$ with a basis of $H_{1}(S)$.

If $C$ is a self-concordance of a knot $J$ in $S^{3}$, then it is also straightforward to see that

$$
H^{2}\left(\left(S^{1} \times S^{3}\right) \backslash N(C)\right) \cong \mathbb{Z}
$$

A generator is given by the Poincaré dual of $\{0\} \times F \subseteq\{0\} \times\left(S^{3} \backslash J\right)$, where $F$ is a Seifert surface of $J$.

Note also that there is a canonical isomorphism

$$
H^{2}\left(B^{4} \backslash N(S), S^{3} \backslash N(K)\right) \cong H^{2}\left(B^{4} \backslash N(S(\gamma, C)), S^{3} \backslash N(K)\right) .
$$

The goal of this section is to prove the following:

Theorem 5.1. Suppose that $S \subseteq B^{4}$ is a properly embedded surface with boundary $K \subseteq S^{3}$, and let $\boldsymbol{\omega}=\left(\omega_{1}, \ldots, \omega_{n}\right)$ denote an $n$-tuple of closed 2-forms in $H^{2}\left(B^{4} \backslash N(S), S^{3} \backslash N(K) ; \mathbb{R}\right)$. Let $\gamma \subseteq S$ be a simple closed curve, and let $T_{\gamma} \subseteq \partial N(S)$ denote the 2-torus which is the preimage of $\gamma$ with respect to the projection $\partial N(S) \rightarrow S$. If $C$ is a self-concordance of a knot $J$, and $S(\gamma, C)$ is the surface obtained by concordance rim surgery on $S$ along $\gamma$ with pattern $C$, then

$$
F_{B^{4}, S(\gamma, C) ; \boldsymbol{\omega}}(1)=\operatorname{Lef}_{Z(\gamma, \boldsymbol{\omega})}(C) \cdot F_{B^{4}, S ; \boldsymbol{\omega}}(1),
$$


where

$$
Z(\gamma, \boldsymbol{\omega})=z_{1}^{\int_{T_{\gamma}} \omega_{1}} \cdots z_{n}^{\int_{T_{\gamma}} \omega_{n}} .
$$

Here, we are giving $S(\gamma, C)$ and $S$ the decoration where the $\mathbf{w}$-subregion is a bigon, and the $\mathbf{z}$ subregion is a genus $g(S)$ subsurface.

We will prove Theorem 5.1 in Section 5.2.

5.1. The Floer homology of the 2-fiber link in $S^{1} \times S^{2}$. In this section, we describe the Floer homology of a simple link in $S^{1} \times S^{2}$ that appears when we do rim surgery. Suppose $S$ is a properly embedded, oriented surface in $B^{4}$, and $\gamma \subseteq S$ is a simple closed curve. Given a normal framing of $S$ along $\gamma$, we may identify a neighborhood of $\gamma$ in $\left(B^{4}, S\right)$ with $\left(S^{1} \times B^{3}, S^{1} \times a\right)$, where $a \subseteq B^{3}$ denotes a properly embedded, unknotted arc. We may view $\left(S^{1} \times B^{3}, S^{1} \times a\right)$ as a link cobordism from $\emptyset$ to

$$
\left(S^{1} \times S^{2}, L_{2}\right),
$$

where $L_{2}$ consists of two $S^{1}$-fibers of $S^{1} \times S^{2}$, with opposite orientations. Let $\mathbb{L}_{2}$ be $L_{2}$ decorated with four basepoints, two on each component. A Heegaard diagram for $\left(S^{1} \times S^{2}, \mathbb{L}_{2}\right)$ is shown in Figure 5.1.

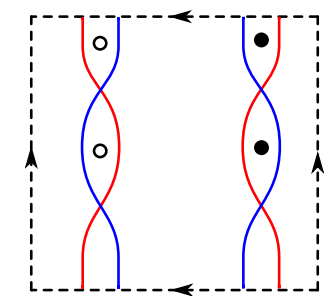

Figure 5.1. A Heegaard diagram for $\left(S^{1} \times S^{2}, \mathbb{L}_{2}\right)$.

Lemma 5.2. The vector space $\widehat{H F L}\left(S^{1} \times S^{2}, \mathbb{L}_{2}\right)$ has rank 4. As a $\left(\mathrm{gr}_{\mathbf{w}}, \mathrm{gr}_{\mathbf{z}}\right)$-bigraded vector space, we have

$$
\widehat{H F L}\left(S^{1} \times S^{2}, \mathbb{L}_{2}\right)=(\mathbb{F})_{(1,-1)} \oplus\left(\mathbb{F}^{2}\right)_{(0,0)} \oplus(\mathbb{F})_{(-1,1)} .
$$

Furthermore, if $\omega$ is any closed 2-form on $\left(S^{1} \times S^{2}\right) \backslash N\left(L_{2}\right)$, then

$$
\widehat{H F L}\left(S^{1} \times S^{2}, \mathbb{L}_{2}, i ; \mathbb{F}[\mathbb{R}]_{\omega}\right) \cong \widehat{H F L}\left(S^{1} \times S^{2}, \mathbb{L}_{2}, i\right) \otimes \mathbb{F}[\mathbb{R}]
$$

for each Alexander grading $i \in \mathbb{Z}$.

Proof. Using the diagram in Figure 5.1, we have

$$
\widehat{C F L}\left(S^{1} \times S^{2}, \mathbb{L}_{2}\right)=(\mathbb{F})_{(1,-1)} \oplus\left(\mathbb{F}^{2}\right)_{(0,0)} \oplus(\mathbb{F})_{(-1,1)} .
$$

The differential vanishes, since there are no index 1 classes that have zero multiplicity at the four basepoints.

Remark 5.3. A basis for $\widehat{H F L}\left(S^{1} \times S^{2}, \mathbb{L}_{2}\right)$ may also be specified using the images of the cobordism maps for $\left(S^{1} \times B^{3}, S^{1} \times a\right)$, with the four dividing sets shown in Figure 5.2. This may be proven using the composition law, and standard TQFT-style arguments, though we will not need this fact. 


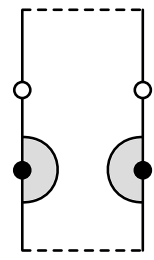

$\mathcal{A}$

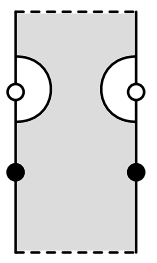

$\overline{\mathcal{A}}$
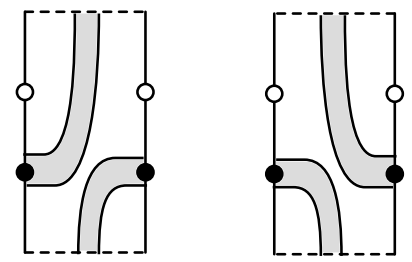

Figure 5.2. Dividing sets on an annulus. The link cobordism maps for $\left(S^{1} \times\right.$ $\left.B^{3}, S^{1} \times a\right)$ with these dividing sets give a basis of $\widehat{H F L}\left(S^{1} \times S^{2}, \mathbb{L}_{2}\right)$.

\subsection{Proof of the concordance rim surgery formula.}

Proof of Theorem 5.1. We factor both link cobordisms through a regular neighborhood of the curve $\gamma$. Consider the annuli

$$
A:=S \cap N(\gamma) \quad \text { and } \quad A^{\prime}:=S(\gamma, C) \cap N(\gamma) .
$$

Let $\mathcal{A}$ and $\mathcal{A}^{\prime}$ denote decorations of $A$ and $A^{\prime}$, respectively, such that the w-subregion consists of two bigons, as on the left side of Figure 5.2. Identify $N(\gamma)$ with $S^{1} \times B^{3}$. We let $\mathcal{W}$ and $\mathcal{W}^{\prime}$ denote the two sutured manifold cobordisms that are complementary to the decorated link cobordisms

$$
\left(S^{1} \times B^{3}, \mathcal{A}\right) \text { and }\left(S^{1} \times B^{3}, \mathcal{A}^{\prime}\right),
$$

respectively.

Write $\boldsymbol{\tau}=\left.\boldsymbol{\omega}\right|_{N(\gamma) \backslash N(S)}$ and $\boldsymbol{\tau}^{\prime}=\boldsymbol{\omega}_{N(\gamma) \backslash N(S(\gamma, C))}$, where $\boldsymbol{\tau}=\left(\tau_{1}, \ldots, \tau_{2 g}\right)$ and $\boldsymbol{\tau}^{\prime}=\left(\tau_{1}^{\prime}, \ldots, \tau_{2 g}^{\prime}\right)$. Using the isomorphism from equation (6), we may assume that

$$
\tau_{i}=\alpha_{i} \cdot P D\left[\{0\} \times F_{U}\right] \text { and } \omega_{i}^{\prime}=\alpha_{i} \cdot P D\left[\{0\} \times F_{K}\right],
$$

for some $\alpha_{i} \in \mathbb{R}$, where $F_{U}$ and $F_{K}$ are Seifert surfaces for the unknot $U$ and for $K$, respectively.

Using the composition law, it suffices to show that

$$
F_{\mathcal{W}^{\prime} ; \boldsymbol{\tau}^{\prime}} \doteq \operatorname{Lef}_{Z(\gamma, \boldsymbol{\omega})}(C) \cdot F_{\mathcal{W} ; \boldsymbol{\tau}}
$$

where $\doteq$ denotes equality up to multiplication by a monomial. Both maps have $\left(\mathrm{gr}_{\mathbf{w}}, \operatorname{gr}_{\mathbf{z}}\right)$-bigrading $(1,-1)$, and hence we can view both maps as having the same rank 1 codomain over $\mathbb{F}\left[\mathbb{R}^{2 g}\right]$ by Lemma 5.2 .

We now consider the link cobordism $\left(S^{1} \times B^{3}, \bar{A}\right)$, obtained by reversing and turning around the orientation of $\mathcal{W}$, which we view as a link cobordism from $\left(S^{1} \times S^{2}, L_{2}\right)$ to the empty set. Write $\overline{\mathcal{A}}$ for $\bar{A}$ decorated with the second dividing set from the left in Figure 5.2. Write $\overline{\mathcal{W}}$ for the sutured manifold cobordism complementary to $\overline{\mathcal{A}}$ in $S^{1} \times B^{3}$, viewed as a cobordism from $\left(S^{1} \times S^{2}, \mathbb{L}_{2}\right)$ to the empty set. We let $\overline{\boldsymbol{\tau}}$ denote the tuple of 2 -forms on $\left(S^{1} \times B^{3}\right) \backslash N(\bar{A})$ which are scalar multiples of the Poincaré dual of $-F_{U}$, as in equation (8).

Since we are viewing the domain and codomain of both $F_{\mathcal{W}^{\prime} ; \boldsymbol{\tau}^{\prime}}$ and $F_{\mathcal{W} ; \boldsymbol{\tau}}$ as rank 1 over $\mathbb{F}\left[\mathbb{R}^{2 g}\right]$, to establish Equation (9), it is sufficient to show that

$$
F_{\overline{\mathcal{W}} ; \bar{\tau}} \circ F_{\mathcal{W}^{\prime} ; \boldsymbol{\tau}^{\prime}} \doteq \operatorname{Lef}_{Z(\gamma, \boldsymbol{\omega})}(C) \cdot F_{\overline{\mathcal{W}} ; \bar{\tau}} \circ F_{\mathcal{W} ; \boldsymbol{\tau}},
$$

and that both sides are non-zero.

The sutured manifold cobordisms $\overline{\mathcal{W}} \circ \mathcal{W}$ and $\overline{\mathcal{W}} \circ \mathcal{W}^{\prime}$ are equal to the complements of the tori $S^{1} \times U$ and $C$ in $S^{1} \times S^{3}$, respectively. Write $\boldsymbol{\omega}_{0}$ for the tuple of 2 -forms which is $\boldsymbol{\tau}$ on $\mathcal{W}$ and $\overline{\boldsymbol{\tau}}$ on $\overline{\mathcal{W}}$. Write $\boldsymbol{\omega}_{0}^{\prime}$ for the tuple of 2 -forms which is $\boldsymbol{\tau}^{\prime}$ on $\mathcal{W}^{\prime}$ and $\overline{\boldsymbol{\tau}}$ on $\overline{\mathcal{W}}$. The proof of [JZ18a, Proposition 5.3] implies that

$$
F_{\overline{\mathcal{W}} \circ \mathcal{W} ; \boldsymbol{\omega}_{0}}(1) \doteq 1 \quad \text { and } \quad F_{\overline{\mathcal{W}} \circ \mathcal{W}^{\prime} ; \boldsymbol{\omega}_{0}^{\prime}}(1) \doteq \operatorname{Lef}_{Z(\gamma, \boldsymbol{\omega})}(C)
$$

which implies equation (10), and hence equation (9), completing the proof. 
Remark 5.4. If we use the alternate definition of concordance rim surgery from Remark 2.2, then one can directly invoke [JZ18a, Corollary 5.5]. Recall that the two definitions are equivalent by the work of Kim [Kim06a, Lemma 2.4] in the case of 1-twist rim surgery.

\section{The invariant $\Omega(S)$}

In this section, we define an invariant $\Omega(S) \in \mathbb{Z}^{\geq 0} \cup\{-\infty\}$ for a surface $S \subseteq B^{4}$ with $g(S)>0$, bounding a knot $K$ in $S^{3}$, and prove that it is a diffeomorphism invariant of $S$.

6.1. Defining $\Omega(S)$. Suppose $R$ is a UFD. We define

$$
\Omega_{R}: R \rightarrow \mathbb{Z}^{\geq 0} \cup\{-\infty\}
$$

to be the number of irreducible (non-unit) factors of an element of $R$, counted with multiplicity. By convention, we set $\Omega(0)=-\infty$.

The map $\Omega$ may be extended to modules, in the following sense. If $M$ is a free, finitely generated $R$-module, then we may define

$$
\Omega_{M}: M \rightarrow \mathbb{Z}^{\geq 0} \cup\{-\infty\},
$$

as follows. We set $\Omega(0)=-\infty$, and, for $x \neq 0$, we set

$$
\Omega_{M}(x)=\max \left\{\Omega_{R}(a): x=a \cdot y, \text { where } a \in R, y \in M\right\} .
$$

Equivalently, we may define $\Omega_{M}(x)$ by picking a free basis $e_{1}, \ldots, e_{n}$ for $M$ over $R$, writing $x=$ $a_{1} e_{1}+\cdots+a_{n} e_{n}$, and setting

$$
\Omega_{M}(x)=\Omega_{M}\left(\operatorname{gcd}\left(a_{1}, \ldots, a_{n}\right)\right) .
$$

If $x \in M$ and $a \in R$, then

$$
\Omega_{M}(a \cdot x)=\Omega_{R}(a)+\Omega_{M}(x) .
$$

Note that, as $\Omega_{M}$ is defined without reference to a basis, it is invariant under $R$-linear isomorphisms of $M$.

We focus now on the group ring $\mathbb{F}\left[\mathbb{R}^{n}\right]$, which is not a UFD. Note that $\mathbb{F}\left[\mathbb{N}^{n}\right]=\mathbb{F}\left[z_{1}, \ldots, z_{n}\right]$ is a UFD. Furthermore, $\mathbb{F}\left[\mathbb{Z}^{n}\right]$ is also a UFD, as it is the localization of $\mathbb{F}\left[\mathbb{N}^{n}\right]$ at the set of monomials. Using the above construction, we obtain a map $\Omega_{\mathbb{F}\left[\mathbb{Z}^{n}\right]}: \mathbb{F}\left[\mathbb{Z}^{n}\right] \rightarrow \mathbb{Z}^{\geq 0} \cup\{-\infty\}$, as well as a similar function for finitely generated, free modules over $\mathbb{F}\left[\mathbb{Z}^{n}\right]$.

Suppose $p \in \mathbb{F}\left[\mathbb{R}^{n}\right]$ is projectively integral (Definition 4.1), and $m$ is a monomial such that $m \cdot p \in$ $\mathbb{F}\left[\mathbb{Z}^{n}\right]$. We then define

$$
\Omega_{\mathbb{F}\left[\mathbb{Z}^{n}\right]}(p):=\Omega_{\mathbb{F}\left[\mathbb{Z}^{n}\right]}(m \cdot p),
$$

which is clearly independent of the choice of monomial $m$.

More generally, suppose $M_{0}$ is a finite dimensional $\mathbb{F}$-vector space, and

$$
M=M_{0} \otimes \mathbb{F}\left[\mathbb{R}^{n}\right] .
$$

If $x \in M$ is projectively integral, and $m \cdot x \in M_{0} \otimes \mathbb{F}\left[\mathbb{Z}^{n}\right]$ for a monomial $m$, we define

$$
\Omega_{M}(x):=\Omega_{M_{0} \otimes \mathbb{F}\left[\mathbb{Z}^{n}\right]}(m \cdot x),
$$

which clearly does not depend on the monomial $m$.

If $f: \mathbb{R}^{n} \rightarrow \mathbb{R}^{n}$ is linear map, then there is an induced endomorphism $\underline{f}$ on the group ring $\mathbb{F}\left[\mathbb{R}^{n}\right]$, defined on monomials via the formula

$$
\underline{f}\left(e^{\mathbf{a}}\right)=e^{f(\mathbf{a})} .
$$

It is straightforward to check the following:

Lemma 6.1. Suppose that $M_{0}$ is an $\mathbb{F}$-vector space and $M=M_{0} \otimes \mathbb{F}\left[\mathbb{R}^{n}\right]$. Suppose further that $\phi$ is an automorphism of $M_{0}$, and $f \in G L_{n}(\mathbb{Z})$. If $x$ is projectively integral, then $(\phi \otimes \underline{f})(x)$ is also projectively integral, and

$$
\Omega_{M}((\phi \otimes \underline{f})(x))=\Omega_{M}(x) .
$$


Definition 6.2. Let $S \subseteq B^{4}$ be an oriented surface of genus $g>0$ in $B^{4}$, bounding a knot $K$ in $S^{3}$, and let $\boldsymbol{\omega}$ be a $2 g$-tuple of integral 2-forms that form a basis of $H^{2}\left(B^{4} \backslash N(S), S^{3} \backslash N(K)\right)$. Let $\mathcal{S}$ denote $S$ decorated with a single dividing arc, such that $g\left(\mathcal{S}_{\mathbf{w}}\right)=0$ and $g\left(\mathcal{S}_{\mathbf{z}}\right)=g(S)$. Proposition 4.3 implies that $\hat{F}_{B^{4}, \mathcal{S} ; \boldsymbol{\omega}}(1)$ is projectively integral. We define

$$
\Omega(S ; \boldsymbol{\omega}):=\Omega_{\widehat{H F K}(K) \otimes \mathbb{F}\left[\mathbb{Z}^{2 g}\right]}\left(\hat{F}_{B^{4}, \mathcal{S} ; \boldsymbol{\omega}}(1)\right) .
$$

6.2. Diffeomorphism invariance of $\Omega$. In this section, we prove that $\Omega(S ; \boldsymbol{\omega})$ is independent of the choice of $\boldsymbol{\omega}$, and furthermore, it is a diffeomorphism invariant of $S$.

Theorem 6.3. Suppose $S$ is an oriented, genus $g>0$ surface bounding a knot $K$ in $S^{3}$. Then the quantity $\Omega(S ; \boldsymbol{\omega}) \in \mathbb{Z}^{\geq 0} \cup\{-\infty\}$ is independent of the choice of integral basis of 2-forms $\boldsymbol{\omega}$, and is a diffeomorphism invariant of the pair $\left(B^{4}, S\right)$.

We begin by considering the dependence of $\Omega(S ; \boldsymbol{\omega})$ on the basis of integral 2 -forms $\boldsymbol{\omega}$ :

Lemma 6.4. Let $S \subseteq B^{4}$ be a properly embedded surface of genus $g>0$, bounding a knot $K$ in $S^{3}$. Suppose that $\boldsymbol{\omega}$ and $\boldsymbol{\omega}^{\prime}$ are two $2 g$-tuples of closed 2-forms that both induce a basis of

$$
G:=H^{2}\left(B^{4} \backslash N(S), S^{3} \backslash N(K) ; \mathbb{R}\right) \cong \mathbb{R}^{2 g},
$$

where $g=g(S)$. Suppose $f: G \rightarrow G$ is an automorphism that sends $[\boldsymbol{\omega}]$ to $\left[\boldsymbol{\omega}^{\prime}\right]$. Using the bases $\boldsymbol{\omega}$ and $\boldsymbol{\omega}^{\prime}$, the map $f$ induces an automorphism of $\mathbb{Z}^{2 g}$, for which we also write $f$. With respect to these identifications, we have

$$
\hat{F}_{B^{4}, \mathcal{S} ; \boldsymbol{\omega}^{\prime}}(1) \doteq\left(\operatorname{id}_{\widehat{H F K}(K)} \otimes \underline{f}^{t}\right)\left(\hat{F}_{B^{4}, \mathcal{S} ; \boldsymbol{\omega}}(1)\right),
$$

where $f^{t}$ denotes the transpose of $f$.

Proof. Write $\boldsymbol{\omega}=\left(\omega_{1}, \ldots, \omega_{2 g}\right)$ and $\boldsymbol{\omega}^{\prime}=\left(\omega_{1}^{\prime}, \ldots, \omega_{2 g}^{\prime}\right)$. Let $\mathcal{W}$ be the sutured manifold cobordism complementary to $S$. Write $f$ as a matrix $\left(a_{i, j}\right)_{1 \leq i, j \leq 2 g}$, such that

$$
\omega_{j}^{\prime}=a_{1, j} \omega_{1}+\cdots+a_{2 g, j} \omega_{2 g}+d \eta_{j},
$$

where $\eta_{j}$ is a 1 -form that vanishes on a neighborhood of $S^{3} \backslash N(K)$.

We consider the totally twisted cobordism map

$$
\underline{F}_{\mathcal{W}}: \mathbb{F} \rightarrow \widehat{H F K}\left(S^{3}, K\right) \otimes_{\mathbb{F}} \mathbb{F}\left[\left(C_{2} / B_{2}\right)\left(B^{4} \backslash N(S)\right)\right],
$$

described in Section 4.

If $(\Sigma, \boldsymbol{\alpha}, \boldsymbol{\beta}, w, z)$ is a Heegaard diagram for $\left(S^{3}, \mathbb{K}\right)$, then we can write

$$
\underline{F}_{\mathcal{W}}(1)=\sum_{\mathbf{x} \in \mathbb{T}_{\alpha} \cap \mathbb{T}_{\beta}} \mathbf{x} \otimes\left(\sum_{i=1}^{n_{\mathbf{x}}} e^{h_{\mathbf{x}, i}}\right),
$$

where $h_{\mathbf{x}, i} \in\left(C_{2} / B_{2}\right)\left(B^{4} \backslash N(S)\right)$, and $n_{\mathbf{x}} \in \mathbb{Z}^{\geq 0}$. The perturbed map $F_{\mathcal{W} ; \boldsymbol{\omega}}$ is given by tensoring with $1 \in \mathbb{F}\left[\mathbb{R}^{2 g}\right]_{\boldsymbol{\omega}}$, so equation (12) becomes

$$
F_{\mathcal{W} ; \boldsymbol{\omega}}(1)=\sum_{\mathbf{x} \in \mathbb{T}_{\alpha} \cap \mathbb{T}_{\beta}} \mathbf{x} \otimes\left(\sum_{i=1}^{n_{\mathbf{x}}} e^{\left(\int_{h_{\mathbf{x}, i}} \omega_{1}, \ldots, \int_{h_{\mathbf{x}, i}} \omega_{2 g}\right)}\right),
$$

and

$$
F_{\mathcal{W} ; \boldsymbol{\omega}^{\prime}}(1)=\sum_{\mathbf{x} \in \mathbb{T}_{\alpha} \cap \mathbb{T}_{\beta}} \mathbf{x} \otimes\left(\sum_{i=1}^{n_{\mathbf{x}}} e^{\left(\int_{h_{\mathbf{x}, i}} \omega_{1}^{\prime}, \ldots, \int_{h_{\mathbf{x}, i}} \omega_{2 g}^{\prime}\right)}\right)
$$

Let us write

$$
f_{0}\left(\omega_{j}\right):=\sum_{i=1}^{2 g} a_{i, j} \omega_{i}
$$


so $f_{0}\left(\omega_{j}\right)=\omega_{j}^{\prime}-d \eta_{j}$. Equation (13) gives

$$
\begin{aligned}
\left(\mathrm{id} \otimes \underline{f}^{t}\right)\left(F_{\mathcal{W} ; \boldsymbol{\omega}}(1)\right) & =\sum_{\mathbf{x} \in \mathbb{T}_{\alpha} \cap \mathbb{T}_{\beta}} \mathbf{x} \otimes\left(\sum_{i=1}^{n_{\mathbf{x}}} e^{f^{t}\left(\int_{h_{\mathbf{x}, i}} \omega_{1}, \ldots, \int_{h_{\mathbf{x}, i}} \omega_{2 g}\right)}\right) \\
& =\sum_{\mathbf{x} \in \mathbb{T}_{\alpha} \cap \mathbb{T}_{\beta}} \mathbf{x} \otimes\left(\sum_{i=1}^{n_{\mathbf{x}}} e^{\left(\int_{h_{\mathbf{x}, i}} f_{0}\left(\omega_{1}\right), \ldots, \int_{h_{\mathbf{x}, i}} f_{0}\left(\omega_{2 g}\right)\right)}\right) .
\end{aligned}
$$

The main claim is that equations (14) and (15) agree up to an overall factor of $e^{\mathbf{a}}$ for some $\mathbf{a} \in \mathbb{R}^{2 g}$. We will show that, if $\mathbf{x} \otimes e^{h}$ and $\mathbf{x}^{\prime} \otimes e^{h^{\prime}}$ are summands of $\underline{F}_{\mathcal{W}}(1)$, then

$$
\int_{h} d \eta_{j}=\int_{h^{\prime}} d \eta_{j}
$$

Indeed, Lemma 4.4 shows that, if $\phi \in \pi_{2}\left(\mathbf{x}, \mathbf{x}^{\prime}\right)$ is a disk on $(\Sigma, \boldsymbol{\alpha}, \boldsymbol{\beta}, w, z)$, then the 2-chain

$$
c:=h-h^{\prime}-\widetilde{\mathcal{D}}(\phi)
$$

is closed, and so $\int_{c} d \eta_{j}=0$. As $\eta_{j}$ vanishes on a neighborhood of $S^{3} \backslash N(K)$, we have

$$
\int_{h} d \eta_{j}=\int_{h^{\prime}} d \eta_{j}
$$

Equation (16) follows, and hence so does the main result.

Combining Lemma 6.1 with Lemma 6.4, we obtain the following:

Corollary 6.5. Let $K$ be a knot in $S^{3}$. If $S$ is a smooth, genus $g>0$ surface in $B^{4}$ bounding $K$, then $\Omega(S ; \boldsymbol{\omega})$ is independent of the integral basis of 2 -forms $\boldsymbol{\omega}$. We henceforth write just $\Omega(S)$.

We now prove that $\Omega(S)$ is a diffeomorphism invariant:

Proof of Theorem 6.3. Suppose that $S$ and $S^{\prime}$ are two genus $g>0$ surfaces in $B^{4}$ that bound a knot $K$ in $S^{3}$, and $\Phi:\left(B^{4}, S\right) \rightarrow\left(B^{4}, S^{\prime}\right)$ is a diffeomorphism. Let $\Phi^{\prime}$ denote the restriction of $\Phi$ to the complement of $S$. Let $\mathcal{S}$ and $\mathcal{S}^{\prime}$ denote $S$ and $S^{\prime}$ decorated with a single dividing arc, with w-subsurfaces both equal to a bigon. Diffeomorphism invariance of the cobordism maps implies that

$$
\hat{F}_{B^{4}, \mathcal{S}^{\prime} ; \Phi_{*}^{\prime} \boldsymbol{\omega}}(1)=\left(\left.\Phi\right|_{\left(S^{3}, K\right) *} \otimes \operatorname{id}_{\mathbb{F}_{\left[\mathbb{R}^{2 g}\right]}}\right)\left(\hat{F}_{B^{4}, \mathcal{S} ; \boldsymbol{\omega}}(1)\right) .
$$

Hence $\Omega\left(S^{\prime}\right)=\Omega(S)$, by Lemma 6.1 .

\subsection{Constructing non-diffeomorphic families of surfaces.}

Theorem 6.6. Suppose that $S \subseteq B^{4}$ is a properly embedded surface, $\gamma \subseteq S$ is a homologically nontrivial simple closed curve, and $C$ is a self-concordance of a knot $J$ that has nontrivial knot Floer Lefschetz polynomial $\operatorname{Lef}_{z}(C)$. If $\hat{F}_{B^{4}, \mathcal{S}} \neq 0$, where $\mathcal{S}$ is a decoration of $S$ such that $\mathcal{S}_{\mathbf{w}}$ is a bigon and $\mathcal{S}_{\mathbf{z}}$ is a genus $g(S)$ subsurface, then $\left(B^{4}, S\right)$ and $\left(B^{4}, S(\gamma, C)\right)$ are not diffeomorphic.

More generally, if $\left\{C_{n}: n \in \mathbb{N}\right\}$ is a set of self-concordances such that $\operatorname{Lef}_{z}\left(C_{n}\right)$ and $\operatorname{Lef}_{z}\left(C_{m}\right)$ have a different number of irreducible factors for $n \neq m$, then $\left(B^{4}, S\left(\gamma, C_{n}\right)\right)$ are pairwise nondiffeomorphic.

Proof. Let $\boldsymbol{\omega}$ denote a collection of closed, integral 2-forms inducing a basis of

$$
G:=H^{2}\left(B^{4} \backslash N(S), S^{3} \backslash N(K) ; \mathbb{R}\right),
$$

and let $\boldsymbol{\omega}^{\prime}$ denote a corresponding basis of integral 2 -forms inducing a basis of

$$
H^{2}\left(B^{4} \backslash N(S(\gamma, C)), S^{3} \backslash N(K) ; \mathbb{R}\right) .
$$

By Theorem 5.1, we have

$$
\hat{F}_{B^{4}, S(\gamma, C) ; \boldsymbol{\omega}^{\prime}}(1)=\operatorname{Lef}_{Z(\gamma, \boldsymbol{\omega})}(C) \cdot \hat{F}_{B^{4}, S ; \boldsymbol{\omega}}(1) .
$$

Since $\gamma$ is homologically non-trivial in $S$, the rim torus $T_{\gamma}$ is homologically non-trivial in $B^{4} \backslash N(S)$. As $\boldsymbol{\omega}$ induces a basis of $G$, the monomial $Z(\gamma, \boldsymbol{\omega})$ is non-constant. Hence, since $\operatorname{Lef}_{z}(C)$ is non-trivial, 
so is $\operatorname{Lef}_{Z(\gamma, \boldsymbol{\omega})}(C)$. Since $\hat{F}_{B^{4}, \mathcal{S}} \neq 0$, we have $\Omega(S) \geq 0$. Hence, Theorem 5.1 and equation (11) imply that if $\operatorname{Lef}_{Z(\gamma, \boldsymbol{\omega})}(C) \neq 1$, then

$$
\Omega(S(\gamma, C))>\Omega(S) \geq 0 .
$$

Theorem 6.3 implies that $S(\gamma, C)$ and $S$ are not diffeomorphic. This completes the proof of the first claim.

The second part follows similarly, as $\Omega\left(S\left(\gamma, C_{n}\right)\right)$ are pairwise distinct.

\section{Quasipositive Knots, ASCEnding surfaces, And Weinstein Cobordisms}

In this section, we provide some background on quasipositive knots, and ascending surfaces in Weinstein cobordisms.

7.1. Quasipositive links and braids. The braid group on $n$ strands has the presentation

$$
\left.B_{n}=\left\langle\sigma_{1}, \ldots, \sigma_{n-1}\right| \sigma_{i} \sigma_{i+1} \sigma_{i}=\sigma_{i+1} \sigma_{i} \sigma_{i+1}, \sigma_{i} \sigma_{j}=\sigma_{j} \sigma_{i} \text { for } i-j \geq 2\right\rangle,
$$

where $\sigma_{i}$ is the braid of a positive crossing between strands $i$ and $i+1$.

A link is positive if it has only positive crossings. A link is quasipositive if it is isotopic to the closure of a braid which can be represented as the product

$$
\mathcal{W}=\prod_{i=1}^{k} w_{i} \sigma_{j_{i}} w_{i}^{-1},
$$

for some $w_{i} \in B_{n}$. Finally, a link is strongly quasipositive if it is the closure of a braid that can be presented as a product of factors of the form

$$
\left(\sigma_{i} \cdots \sigma_{j-2}\right)\left(\sigma_{j-1}\right)\left(\sigma_{i} \cdots \sigma_{j-2}\right)^{-1}
$$

for $i \leq j-2$. Rudolph [Rud99] proved that all positive links are strongly quasipositive.

If $K$ is a quasipositive link, which is the closure of a word $\mathcal{W}$ with presentation as in equation (19), then there is an induced link cobordism from the empty link to $K$. The link cobordism starts with $n$ births, which give an unlink of $n$ components, which we view as the closure of a trivial $n$-stranded braid. Each factor of the form $w_{i} \sigma_{j_{i}} w_{i}^{-1}$ determines an isotopy, followed by a saddle. We call this the quasipositive link cobordism of the word $\mathcal{W}$.

If each factor of $\mathcal{W}$ has the form of equation (20), then it gives rise to a Seifert surface for $K$ that we call the quasipositive Seifert surface of the word $\mathcal{W}$. This is isotopic to the quasipositive link cobordism of $\mathcal{W}$ relative to $K$.

7.2. Weinstein manifolds and ascending surfaces. In this section, we recall background on Weinstein manifolds and a natural family of smooth embedded surfaces therein, called ascending surfaces. See [CE12] for additional background. Our exposition mostly follows Hayden [Hay17].

Definition 7.1. If $(X, J)$ is a complex manifold, we say that a map $\phi: X \rightarrow \mathbb{R}$ is $J$-convex if the 2 -form $\omega_{\phi}:=-d\left(d^{\mathbb{C}} \phi\right)$ is symplectic, where $d^{\mathbb{C}} \phi=d \phi \circ J$. A compact, complex manifold $(X, J)$ is called Stein if it admits a $J$-convex function which is exhausting (i.e., proper and bounded from below). If $X$ is compact and has boundary $-Y_{0} \cup Y_{1}$, we say that $(X, J)$ is a Stein cobordism from $Y_{0}$ to $Y_{1}$ if it admits a $J$-convex function $\phi: X \rightarrow[0,1]$ such that 0 and 1 are regular values, and $\phi^{-1}(0)=Y_{0}$ and $\phi^{-1}(1)=Y_{1}$. Then $\xi_{i}:=T Y_{i} \cap J\left(T Y_{i}\right)$ is a contact structure on $Y_{i}$ for $i \in\{0,1\}$.

Definition 7.2. A Weinstein manifold is a tuple $(X, \omega, \phi, V)$, where $(X, \omega)$ is a symplectic manifold, $\phi$ is an exhausting Morse function, and $V$ is a Liouville vector field (i.e., $\mathcal{L}_{V} \omega=\omega$ ) which is gradientlike for $\phi$.

If $(X, J)$ is a Stein manifold with $J$-convex Morse function $\phi$, then $\left(X, \omega_{\phi}, \phi, V_{\phi}\right)$ is a Weinstein manifold, where $V_{\phi}$ is the gradient of $\phi$ with respect to the metric $\langle X, Y\rangle:=\omega_{\phi}(X, J Y)$. We will be interested in link cobordisms in Weinstein manifolds which satisfy the following property. 
Definition 7.3. A smoothly embedded surface $S$ in a Weinstein manifold $(X, \omega, \phi, V)$ is called ascending if $S$ contains no critical points of $\phi$, the restriction $\left.\phi\right|_{S}$ is Morse, and $\left.d \phi \wedge i_{V} \omega\right|_{S}>0$ away from the critical points of $\left.\phi\right|_{S}$.

The definition is due to Boileau-Orevkov [BO01, Définition 2] when $X=B^{4}$, and Hayden [Hay17, Definition 4.1] for general Stein and Weinstein $X$. We could equivalently require each regular level set $\left.\phi\right|_{S} ^{-1}(c)$ to be a positive transverse link in the hypersurface $\phi^{-1}(c)$ with the contact form $i_{V} \omega$.

The tangent spaces at the critical points of ascending surfaces in Stein manifolds are always $J$ complex lines; see Boileau-Orevkov [BO01, p. 828] and Hayden [Hay17, Proposition 4.10]. Hayden's proof adapts to show that if $p \in S$ is a critical point of $\left.\phi\right|_{S}$, then $i_{V} \omega$ restricts trivially to $T_{p} S$, which implies that $\omega$ restricts non-trivially to $\Lambda^{2} T_{p} S$.

Definition 7.4. If $S$ is an ascending surface in a Weinstein manifold $(W, \omega, \phi, V)$, we say that a critical points $p \in S$ of $\left.\phi\right|_{S}$ is positive (resp. negative) if the symplectic form restricts positively (resp. negatively) to $T_{p} S$.

If $S$ is an ascending surface in a Stein manifold $(X, J)$ with $J$-convex Morse function $\phi$, then a critical point $p \in S$ of $\left.\phi\right|_{S}$ is positive precisely when the orientation of $T_{p} S$ coincides with the complex orientation from $J$. If $S$ is a $J$-holomorphic curve in a Stein manifold $(X, J)$, then $S$ is an ascending surface with respect to a generic $J$-convex $\phi$ [Hay17, Proposition 4.11].

The following lemma seems to be well-known; however, we have been unable to locate a reference.

Lemma 7.5. Suppose that $(X, \omega, \phi, V)$ is Weinstein. Suppose further that $S \subseteq X$ is an ascending surface and $p$ is a critical point of $\left.\phi\right|_{S}$. If $p$ has index 0 , then $p$ is positive. If $p$ has index 2 , then $p$ is negative.

Proof. We focus on the case when $p$ has index 0 , since the argument is symmetric when $p$ has index 2 . Let $\epsilon>0$ be small. Note that shifting $\phi$ by an overall constant preserves the Weinstein condition on $\phi$, and the ascending condition on $S$, so assume $\phi(p)=-\epsilon$. Let $D_{\epsilon} \subseteq S$ denote the component of $\left.\phi\right|_{S} ^{-1}([-\epsilon, 0])$ containing $p$. Note that $\phi=0$ on $\partial D_{\epsilon}$.

Recall that the ascending condition on $S$ is equivalent to $\left.\phi\right|_{S}$ being Morse, and $\left.d \phi \wedge i_{V} \omega\right|_{S}>0$ away from the critical points. As we saw above, an easy adaptation of [Hay17, Proposition 4.5] gives that $\omega$ restricts non-trivially to $T_{p} S$. Hence, it suffices to compute the sign.

Since $\phi=0$ on $\partial D_{\epsilon}$, Stoke's theorem gives

$$
\int_{D_{\epsilon}} d\left(\phi i_{V} \omega\right)=\int_{\partial D_{\epsilon}} \phi i_{V} \omega=0 .
$$

On the other hand, the Leibniz rule gives

$$
d\left(\phi i_{V} \omega\right)=d \phi \wedge i_{V} \omega+\phi \omega,
$$

since $d i_{V} \omega=\omega$ by $\mathcal{L}_{V} \omega=\omega$. Hence, combining equations (21) and (22), we obtain

$$
0=\int_{D_{\epsilon}} d\left(\phi i_{V} \omega\right)=\int_{D_{\epsilon}} d \phi \wedge i_{V} \omega+\int_{D_{\epsilon}} \phi \omega .
$$

Since $\left.d \phi \wedge i_{V} \omega\right|_{S}>0$ away from $p$, we conclude that $\int_{D_{\epsilon}} \phi \omega<0$. Since $\phi \leq 0$ near $p$, we conclude that $\omega$ must be positive near $p$.

7.3. Open books and transverse knots. We begin with some background on open books and transverse knots in contact manifolds.

Definition 7.6. $(\mathcal{O}-1)$ An abstract open book $\mathcal{O}$ is a pair $(F, h)$, where $F$ is a compact, oriented surface with boundary, and $h: F \rightarrow F$ is an automorphism such that $\left.h\right|_{\partial F}=$ id. Note that $\mathcal{O}$ determines a 3 -manifold $Y_{\mathcal{O}}=(F \times I) / \sim$, where $(x, 1) \sim(h(x), 0)$ for all $x \in F$, and $(x, t) \sim\left(x, t^{\prime}\right)$ whenever $t, t^{\prime} \in I$ and $x \in \partial F$.

$(\mathcal{O}-2)$ An embedded open book $(F, h, \phi)$ of $Y$ consists of an abstract open book $\mathcal{O}=(F, h)$, and a diffeomorphism

$$
\varphi: Y_{\mathcal{O}} \rightarrow Y \text {. }
$$


We say that $(F, h, \phi)$ is compatible with a co-oriented contact structure $\xi$ if $\xi$ is isotopic to a contact structure which is the kernel of a 1-form $\alpha$ such that $d \alpha>0$ on the interior of the pages of the open book, and $\alpha>0$ on the binding.

Definition 7.7. $(\mathcal{T}-1)$ Suppose that $(Y, \xi)$ is a co-oriented contact 3-manifold. A link $L$ in $(Y, \xi)$ is transverse if $T_{p} L$ is transverse to $\xi_{p}$ for all $p \in L$. If $L$ is oriented, we say that $L$ is a positive transverse link if $L$ is positively transverse to $\xi$.

$(\mathcal{T}-2)$ An abstract, pointed open book $\mathcal{O}$ is a tuple $(F, h, \mathbf{p})$, where $(F, h)$ is an open book, and $\mathbf{p} \subseteq \operatorname{int}(F)$ is a finite collection of points which is fixed by $h$ setwise. Note that a pointed open book determines a 3-manifold $Y_{\mathcal{O}}$ containing a link $L_{\mathcal{O}}$.

$(\mathcal{T}$-3) If $\mathbb{L}=(L, \mathbf{w}, \mathbf{z})$ is a multi-pointed link in a 3-manifold $Y$, we say an embedded, pointed open book of $(Y, \mathbb{L})$ is a tuple $(F, h, \mathbf{p}, \phi)$ such that $(F, h, \phi)$ is an embedded open book for $Y$, and

(a) $\varphi(\mathbf{p} \times I)=L$,

(b) $\varphi(\mathbf{p} \times\{1 / 2\})=\mathbf{w}$ and $\varphi(\mathbf{p} \times\{0\})=\mathbf{z}$.

$(\mathcal{T}$-4) If $\mathbb{L}=(L, \mathbf{w}, \mathbf{z})$ is a multi-pointed, transverse link in $(Y, \xi)$, we say that an embedded, pointed open book for $(Y, \mathbb{L})$ is compatible with $\xi$ if there is an isotopy of contact 1-forms $\alpha_{t}$ such that

(a) $\xi=\operatorname{ker} \alpha_{0}$,

(b) $\alpha_{t}>0$ on $L$ for each $t$,

(c) $d \alpha_{1}>0$ on the pages, and $\alpha_{1}>0$ on the binding.

Definition 7.8. Suppose that $\mathcal{O}=(F, h, \mathbf{p})$ is a pointed open book. A basis of arcs of $\mathcal{O}$ consists of a collection of pairwise disjoint $\operatorname{arcs} \mathbf{a}=\left(a_{1}, \ldots, a_{n}\right)$, such that each component of $F \backslash \mathbf{a}$ is a disk containing exactly one point of $\mathbf{p}$.

7.4. Partial open books and transverse knots. In this section, extending work of Stipsicz and Vértesi [SV09] for doubly-pointed transverse knots, we construct a contact structure on the sutured manifold complementary to a multi-pointed transverse link by attaching bypasses to the complement of a Legendrian approximation, and relate the corresponding partial open books. We will use this for showing the naturality of the transverse link invariants in Section 8.2.

The following definition is due to Honda, Kazez, and Matić [HKM09]:

Definition 7.9. An abstract partial open book $\mathcal{O}$ is a triple $\mathcal{O}=(F, P, h)$, where $F$ is a compact, connected, oriented surface with non-empty boundary, $P \subseteq F$ is a compact subsurface such that $F$ is obtained from $\operatorname{cl}(F \backslash P)$ by successively attaching 1-handles, and $h: P \rightarrow F$ is an embedding such that $\left.h\right|_{P \cap \partial F}=\mathrm{id}$.

A partial open book $\mathcal{O}$ naturally determines a 3-manifold manifold $M_{\mathcal{O}}=(F \times I) / \sim$, where $(x, 1) \sim(h(x), 0)$ for $x \in P$, and $(x, t) \sim\left(x, t^{\prime}\right)$ if $x \in \partial F \cap \partial P$ and $t, t^{\prime} \in I$, with sutures $\gamma_{\mathcal{O}}=\partial(F \backslash P) \times\{1\}$. If $(M, \gamma)$ is a sutured manifold, then an embedded partial open book is a partial open book $\mathcal{O}$ equipped with a diffeomorphism $\varphi:\left(M_{\mathcal{O}}, \gamma_{\mathcal{O}}\right) \rightarrow(M, \gamma)$.

If $\mathbb{L}=(L, \mathbf{w}, \mathbf{z})$ is a multi-pointed link in $Y$, we will write $Y(\mathbb{L})$ for the complementary sutured manifold obtained by removing a neighborhood of $L$, and adding meridional sutures corresponding to the basepoints of $\mathbb{L}$.

Suppose that $\mathbb{L}=(L, \mathbf{w}, \mathbf{z})$ is a multi-pointed transverse link in $(Y, \xi)$. Let $\mathcal{L}$ be a Legendrian approximation of $L$. Choose a standard neighborhood $N(\mathcal{L})$ of $\mathcal{L}$, and write $\xi_{0}=\left.\xi\right|_{Y \backslash N(\mathcal{L})}$. The dividing set on $\partial(Y \backslash N(\mathcal{L}))$ consists of two copies of the contact longitude on each component of $L$.

Extending the construction of Stipsicz and Vértesi [SV09] from doubly-pointed to multi-pointed knots, we attach a collection of bypasses to $\xi_{0}$ along $\partial(Y \backslash N(\mathcal{L}))$ to obtain a contact structure $\xi_{\mathbb{L}}^{\text {巾 }}$ on $Y(\mathbb{L})$. If $K$ is a component of $\mathbb{L}$ that has $2 n$ basepoints, then we attach $n$ bypasses along $\partial N(K)$, as in Figure 7.1.

Following the proof of [SV09, Theorem 1.5], we see that the contact structure $\xi_{\mathbb{L}}^{\text {त }}$ on $Y \backslash N(L)$ is invariant under negative stabilizations of $\mathcal{L}$, up to isotopy relative to $\partial(Y \backslash N(L))$, in the following sense. If $\mathcal{L}^{-}$is a negative stabilization, we may view $\mathcal{L}^{-}$as lying in $N(L)$. Under the canonical diffeomorphism between $Y \backslash N(\mathcal{L})$ and $Y \backslash N\left(\mathcal{L}^{-}\right)$, the contact structures constructed from $\mathcal{L}$ and $\mathcal{L}^{-}$are isotopic, relative to $\partial(Y \backslash N(L))$. 


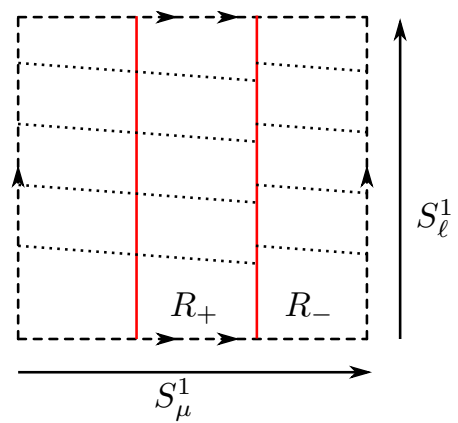

FiguRE 7.1. Bypass attachments along $\partial(Y \backslash N(L))$ to obtain $\xi_{\mathbb{L}}^{\pitchfork}$ from $\xi_{0}$.

Given a pointed open book $\mathcal{O}=(F, \mathbf{p}, h)$ for a multi-pointed link $\mathbb{L}$ in $Y$, we may obtain a partial open book $\mathcal{O}^{\circ}:=\left(F^{\circ}, P,\left.h\right|_{P}\right)$ for the sutured manifold $Y(\mathbb{L})$, as follows. Take $F^{\circ}=F \backslash N(\mathbf{p})$. The subsurface $P$ is obtained from $F^{\circ}$ by removing a neighborhood of $|\mathbf{p}|$ pairwise disjoint arcs, each connecting a point of $\mathbf{p}$ to $\partial F$. The partial open book $\mathcal{O}^{\circ}$ is clearly a partial open book for $Y(\mathbb{L})$. Moreover, we have the following:

Proposition 7.10. Let $\mathbb{L}$ be a multi-pointed transverse link in the closed contact 3-manifold $(Y, \xi)$. If $(\mathcal{O}, \varphi)$ is an embedded, pointed open book for $(Y, \mathbb{L})$ compatible with $\xi$ in the sense of part $(\mathcal{T}-4)$ of Definition 7.7, then the partial open book $\left(\mathcal{O}^{\circ},\left.\varphi\right|_{Y_{\mathcal{O}^{\circ}}}\right)$ for $Y(\mathbb{L})$ is compatible with $\xi_{\mathbb{L}}^{\text {. }}$.

Proof. The proof is by induction on the number of basepoints per component. The case when each component has two basepoints follows from the work of Stipsicz and Vértesi [SV09]. To obtain the statement more generally, we argue by induction. To increase the number of basepoints on a pointed open book, we may perform a positive Markov stabilization to $\mathcal{O}$, as described by Baldwin, Vela-Vick, and Vértesi [BVVV13, Section 2.5]; see the left of Figure 8.6. To see that this has the same effect as one of our bypass attachments, we argue as follows. Bypasses may be attached one at a time. In Figure 7.2, we illustrate one of the attaching arcs of a bypass used in the construction of $\xi_{\mathbb{L}}^{\pitchfork}$, after one bypass has already been attached. A bypass attachment is, by definition, a contact 1-handle followed by a contact 2-handle. The feet of the contact 1-handle are attached at the end points of the bypass arc, and the contact 2-handle is attached along the union of a longitude of the 1-handle, and the original bypass arc. The effect of contact 1-handles and 2-handles on partial open books is described in [JZ18b, Figure 1.1]. An easy local computation shows the effect on partial open books of the bypass shown on the right-hand side of Figure 7.2 coincides with a positive Markov stabilization of pointed open books.

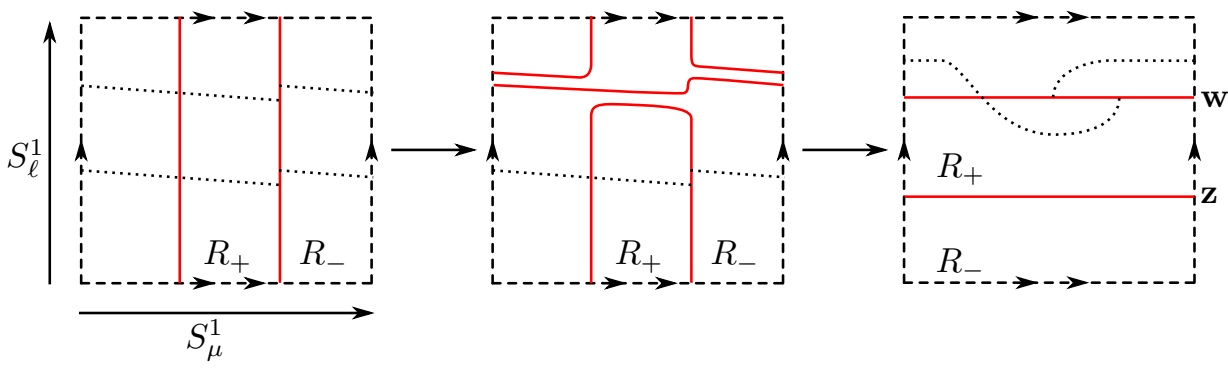

Figure 7.2. Left: Two bypasses giving $\xi_{\mathbb{L}}^{\text {}}$. Middle: The effect of attaching the top bypass. Right: An isotopy of the middle picture. The sutures on the right are labeled by the corresponding basepoint. 
7.5. Ascending surfaces and open books. In this section, we recall how to interpret ascending surfaces in terms of pointed open books. Given a contact manifold $(Y, \xi)$, we denote its symplectization by $\operatorname{Symp}(Y, \xi)$. We first consider index 0 critical points of an ascending surface, which are always positive by Lemma 7.5.

Lemma 7.11. Suppose that $(W, \mathcal{S}):\left(Y, \mathbb{L}_{0}, \xi\right) \rightarrow\left(Y, \mathbb{L}_{1}, \xi\right)$ is an ascending link cobordism with $W=[a, b] \times Y \subseteq \operatorname{Symp}(Y, \xi)$, such that $\mathcal{S}$ has a single (positive) index 0 critical point $p$, and $\mathcal{S}$ is locally translation invariant outside of a small neighborhood of $p$, and the basepoints of $\mathbb{L}_{1}$ are the images of the basepoints of $\mathbb{L}_{0}$ under the translation in the $[a, b]$-direction. If $\mathcal{O}=(F, \mathbf{p}, h)$ is a pointed open book for $\left(Y, \mathbb{L}_{0}, \xi\right)$, then a pointed open book for $\left(Y, \mathbb{L}_{1}, \xi\right)$ may be obtained (after an ambient isotopy of $\mathcal{O}$ ) by adding a basepoint near the boundary of $F$, and keeping $h$ unchanged.

Proof. Suppose $(c, p) \in[a, b] \times Y$ is the critical point of $\mathcal{S}$. Isotope $\mathcal{O}$ so that the binding passes through $p$, transverse to $\xi_{p}$. Then the regular level sets of $\mathcal{S}$ immediately after $c$ intersect the pages of the open book in a single point, since $T_{(c, p)} S=\{c\} \times \xi_{p}$. Let the intersection of the level set of $S$ with value $c+\epsilon$ and a page of the open book be the new basepoint. It is straightforward to see that this open book has the desired properties.

We now consider index 1 critical points. We recall that, if $p_{1}$ and $p_{2}$ are distinct points on a surface $\Sigma$ connected by an embedded path $\gamma$, there are half-twist diffeomorphisms tw ${ }_{\gamma}^{ \pm}$that swap $p_{1}$ and $p_{2}$, and are supported in a neighborhood of $\gamma$, as in Figure 7.3.

Lemma 7.12. Suppose that $(W, \mathcal{S}):\left(Y, \mathbb{L}_{0}, \xi\right) \rightarrow\left(Y, \mathbb{L}_{1}, \xi\right)$ is an ascending link cobordism with $W=[a, b] \times Y \subseteq \operatorname{Symp}(Y, \xi)$, such that $\mathcal{S}$ has a single index 1 critical point, of sign $\epsilon= \pm 1$. Suppose further that, outside a small neighborhood of the critical point, the surface $\mathcal{S}$ is locally translation invariant, and the basepoints of $\mathbb{L}_{1}$ coincide with the images of the basepoints of $\mathbb{L}_{0}$ under translation. Then we may pick a pointed open book $(F, \mathbf{p}, h)$ for $\left(Y, \mathbb{L}_{0}, \xi\right)$ such that $\left(F, \mathbf{p}, \mathrm{tw}_{\gamma}^{\epsilon} \circ h\right)$ is a pointed open book for $\left(Y, \mathbb{L}_{1}, \xi\right)$, where $\mathrm{tw}_{\gamma}^{\epsilon}$ is a half-twist diffeomorphism associated to a path on $F$ connecting two distinct basepoints, of sign $\epsilon$.

A proof of Lemma 7.12 may be found by following the proof of [Hay17, Theorem 4.3]; see specifically Lemma 3.5 therein.
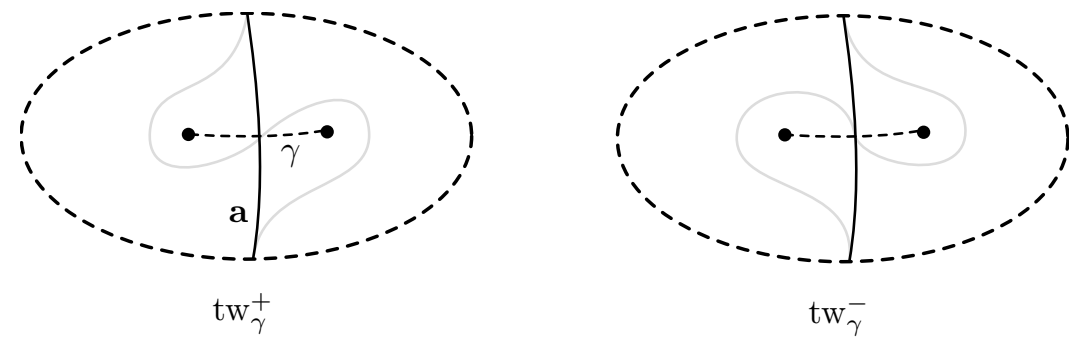

FIGURE 7.3. The positive and negative half-twist diffeomorphisms.

\section{TRANSVERSE INVARIANTS, AND THEIR FUnCTORIALITY UNDER ASCENDING WEINSTEIN COBORDISMS}

Lisca, Ozsváth, Stipsicz, and Szabó [LOSS09] described an invariant of Legendrian knots, which is commonly referred to as the LOSS invariant. The LOSS invariant is unchanged by negative stabilizations, and hence gives an invariant of transverse knots, by work of Epstein, Fuchs, and Meyer [EFM01] and Etnyre and Honda [EH01]. Baldwin, Vela-Vick, and Vértesi [BVVV13] constructed an extension of the LOSS invariant using multi-pointed open books that they called the BRAID invariant, which is more immediately an invariant of transverse links. We will refer to the common invariant as the transverse invariant. If $\mathbb{L}=(L, \mathbf{w}, \mathbf{z})$ is a transverse, multi-pointed link in the contact 3-manifold $(Y, \xi)$, the transverse invariants take the form of elements

$$
\hat{\mathfrak{T}}(Y, \mathbb{L}, \xi) \in \widehat{H F L}(-Y,-\mathbb{L}) \quad \text { and } \quad \mathfrak{T}^{-}(Y, \mathbb{L}, \xi) \in H F L^{-}(-Y,-\mathbb{L}) .
$$


See Remark 8.2 for a note on orientation conventions. We will review the construction of the transverse invariant in Section 8.1.

In this section, we study the functoriality of the transverse invariants. We restate our main result, Theorem 1.2 from the introduction, after the following definition.

Definition 8.1. Suppose $(W, \mathcal{S}):\left(Y_{0}, \mathbb{L}_{0}\right) \rightarrow\left(Y_{1}, \mathbb{L}_{1}\right)$ is a decorated link cobordism, equipped with a Morse function $\phi: S \rightarrow I$. Write $\mathbb{L}_{i}=\left(L_{i}, \mathbf{w}_{i}, \mathbf{z}_{i}\right)$. We say $\mathcal{S}$ is $\mathbf{w}$-arboreal with respect to $\phi$ if there is a forest $F \subseteq \mathcal{S}_{\mathbf{w}}$ (i.e., a disjoint union of trees) such that the following hold:

(1) $\mathcal{S}_{\mathbf{w}}$ is a regular neighborhood of $F$.

(2) $d \phi$ restricts non-trivially to each edge of $F$.

(3) Each vertex $v$ of $F \cap \operatorname{int}(W)$ is incident to exactly one edge on which $\phi$ takes lower value than $\phi(v)$.

We say that a decorated link cobordism $(W, \mathcal{S}):\left(Y_{0}, \mathbb{L}_{0}\right) \rightarrow\left(Y_{1}, \mathbb{L}_{1}\right)$ is w-anti-arboreal with respect to a Morse function $\phi: S \rightarrow I$ if the reversed link cobordism $(W, \mathcal{S})^{\vee}:\left(-Y_{1},-\mathbb{L}_{1}\right) \rightarrow\left(-Y_{0},-\mathbb{L}_{0}\right)$ is $\mathbf{w}$-arboreal with respect to $-\phi$. Note that the composition of $\mathbf{w}$-arboreal decorations is $\mathbf{w}$-arboreal. See Figure 8.1 for an example of a w-anti-arboreal decoration.

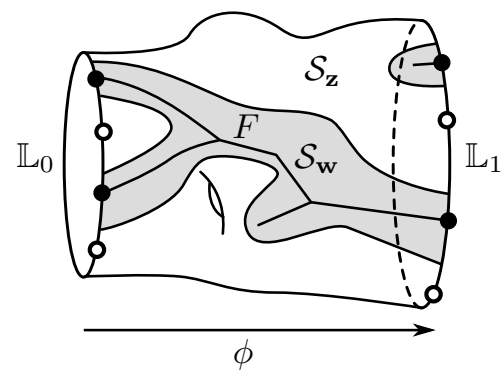

FiguRE 8.1. A w-anti-arboreal decoration of a cobordism from $\mathbb{L}_{0}$ to $\mathbb{L}_{1}$.

The main theorem of this section is the following:

Theorem 1.2. Suppose $(W, \mathcal{S}):\left(Y_{0}, \mathbb{L}_{0}\right) \rightarrow\left(Y_{1}, \mathbb{L}_{1}\right)$ is a decorated link cobordism such that $W$ has a Weinstein structure $(W, \omega, \phi, V)$, and $\mathcal{S}=(S, \mathcal{A})$, where $S$ is an ascending surface with positive critical points. If the decoration $\mathcal{A}$ is $\mathbf{w}$-anti-arboreal with respect to $\phi$, then

$$
\left(F_{W, \mathcal{S}}^{\circ}\right)^{\vee}\left(\mathfrak{T}^{\circ}\left(\mathbb{L}_{1}\right)\right)=\mathfrak{T}^{\circ}\left(\mathbb{L}_{0}\right),
$$

for $\circ \in\{\wedge,-\}$.

Remark 8.2. Our orientation convention in equation (23) departs slightly from the conventions of Lisca, Ozsváth, Stipsicz, and Szabó [LOSS09], and Baldwin, Vela-Vick, and Vértesi [BVVV13], where the transverse invariants lie in $H_{F} L^{\circ}(-Y, \mathbb{L})$. Note, however, that there is an isomorphism $H F L^{\circ}(-Y, \mathbb{L}) \cong H F L^{\circ}(-Y,-\mathbb{L})$. Ozsváth, Szabó, and Thurston [OST08] define two transverse invariants, denoted $\lambda^{+}$and $\lambda^{-}$. The invariant $\lambda^{+}$lies in $H F L^{\circ}(-Y, \mathbb{L})$, and coincides with the LOSS/BRAID invariants, as defined in [LOSS09] and [BVVV13], according to [BVVV13, Theorem 8.1]. The invariant $\lambda^{-}$lies in $\operatorname{HFL}^{\circ}(-Y,-\mathbb{L})$, and coincides with the invariant for which we write $\mathfrak{T}^{\circ}(Y, \mathbb{L}, \xi)$. Theorem 1.2 could be stated for the invariants in $H F L^{\circ}(-Y, \mathbb{L})$ by considering $\mathbf{z}$-anti-arboreal decorations and the cobordism map $F_{W,-\mathcal{S}}^{\circ}$, where $-\mathcal{S}$ denotes $\mathcal{S}$ with the opposite orientation.

We have the following immediate corollaries of Theorem 1.2:

Corollary 8.3. Let the knot $K$ in $S^{3}$ be the closure of a quasipositive braid, and let $S$ be the corresponding quasipositive link cobordism. We decorate $\mathcal{S}$ with a dividing set consisting of a single arc, so that $g\left(\mathcal{S}_{\mathbf{w}}\right)=0$ and $g\left(\mathcal{S}_{\mathbf{z}}\right)=g(S)$. We view $\left(B^{4}, \mathcal{S}\right)$ as a cobordism from $\emptyset$ to $\left(S^{3}, K\right)$. As $K$ is in braid position, it is a transverse knot in the standard tight contact structure on $S^{3}$. We have

$$
\left(\hat{F}_{B^{4}, \mathcal{S}}\right)^{\vee}(\hat{\mathfrak{T}}(K))=1 \in \widehat{H F K}(\emptyset) \cong \mathbb{F}_{2} \quad \text { and } \quad\left(F_{B^{4}, \mathcal{S}}^{-}\right)^{\vee}\left(\mathfrak{T}^{-}(K)\right)=1 \in H F K^{-}(\emptyset) \cong \mathbb{F}[v] .
$$


Corollary 8.4. Suppose that $\mathcal{S} \subseteq B^{4}$ is a decorated surface obtained by pushing a quasipositive Seifert surface of a knot $K$ into $B^{4}$, with a decoration such that $g\left(\mathcal{S}_{\mathbf{w}}\right)=0$ and $g\left(\mathcal{S}_{\mathbf{z}}\right)=g(S)$. Then

$$
F_{B^{4}, \mathcal{S}}^{\circ}: \operatorname{HFK}^{\circ}(\emptyset) \rightarrow \operatorname{HFK}^{\circ}(K)
$$

are non-vanishing, for $\circ \in\{\wedge,-\}$.

We now state a stronger version of Theorem 1.2 for the cobordism maps with perturbed coefficients, which we will prove at the end of Section 8.6.

Proposition 8.5. Suppose that $(W, \mathcal{S}):\left(Y_{0}, \mathbb{L}_{0}\right) \rightarrow\left(Y_{1}, \mathbb{L}_{1}\right)$ is a decorated link cobordism, with $W$ Weinstein, and $\mathcal{S}$ an ascending surface with positive critical points and $\mathbf{w}$-anti-arboreal decoration. Suppose further that $\boldsymbol{\omega}$ is an $n$-tuple of closed 2-forms on the complement of $\mathcal{S}$ in $W$ that vanish on $Y_{0}$ and $Y_{1}$. Then,

$$
\left(\hat{F}_{W, \mathcal{S} ; \omega}\right)^{\vee}\left(\hat{\mathfrak{T}}\left(\mathbb{L}_{1}\right) \otimes 1\right) \doteq \hat{\mathfrak{T}}\left(\mathbb{L}_{0}\right) \otimes 1,
$$

as elements of $\widehat{H F L}\left(-Y_{0},-\mathbb{L}_{0}\right) \otimes \mathbb{F}\left[\mathbb{R}^{n}\right]$. In particular, if $K \subseteq S^{3}$ is the closure of a quasipositive braid and $S \subseteq B^{4}$ is the associated ascending surface, then $\Omega(S)=0$.

8.1. The transverse invariants. We now review the definition of the transverse invariants, following Baldwin, Vela-Vick, and Vértesi [BVVV13]. Suppose that $\mathbb{L}=(L, \mathbf{w}, \mathbf{z})$ is a multi-pointed, transverse link in the contact 3-manifold $(Y, \xi)$. We pick an embedded, pointed open book $\mathcal{O}=(S, h, \mathbf{p})$ for $(Y, \mathbb{L}, \xi)$. We construct a multi-pointed link diagram

$$
\mathcal{H}(\mathcal{O}):=(\Sigma, \boldsymbol{\alpha}, \boldsymbol{\beta}, \mathbf{w}, \mathbf{z})
$$

for $(Y, \mathbb{L})$, as follows. We define

$$
\Sigma:=F \times\{1 / 2\} \cup \bar{F} \times\{0\} .
$$

Let $\mathbf{a}=\left\{a_{1}, \ldots, a_{n}\right\}$ be a basis of $\operatorname{arcs}$ for $\mathcal{O}$, as in Definition 7.8. For $i \in\{1, \ldots, n\}$, let $b_{i}$ be a small translate of $a_{i}$ such that we move $\partial a_{i}$ positively along $\partial F$, and such that there is a single transverse intersection point between $a_{i}$ and $b_{i}$. We define

$$
\alpha_{i}:=a_{i} \times\{1 / 2\} \cup a_{i} \times\{0\} \quad \text { and } \quad \beta_{i}:=b_{i} \times\{1 / 2\} \cup h\left(b_{i}\right) \times\{0\} .
$$

Furthermore, let $\mathbf{w}=\mathbf{p} \times\{1 / 2\} \in F \times\left\{\frac{1}{2}\right\}$ and $\mathbf{z}=\mathbf{p} \times\{0\} \in \bar{F} \times\{0\}$. Finally, set

$$
\mathcal{H}^{\vee}(\mathcal{O}):=(\Sigma, \boldsymbol{\beta}, \boldsymbol{\alpha}, \mathbf{w}, \mathbf{z}),
$$

which is a diagram for $(-Y,-\mathbb{L})$.

For $\circ \in\{\wedge,-\}$, the transverse invariants

$$
\hat{\mathfrak{T}}(Y, \mathbb{L}, \xi) \in \widehat{H F L}\left(\mathcal{H}^{\vee}(\mathcal{O})\right) \quad \text { and } \quad \mathfrak{T}^{-}(Y, \mathbb{L}, \xi) \in H F L^{-}\left(\mathcal{H}^{\vee}(\mathcal{O})\right)
$$

are given by the homology class of the intersection point

$$
\mathbf{x}(\mathcal{O}):=\left(x_{1}, \ldots, x_{n}\right)
$$

where $x_{i}=a_{i} \times\{1 / 2\} \cap b_{i} \times\{1 / 2\}$. See Figure 8.2 for an example of a planar open book $\mathcal{O}$, the Heegaard diagram $\mathcal{H}^{\vee}(\mathcal{O})$, and the intersection point $\mathbf{x}(\mathcal{O})$.

8.2. Naturality of the transverse invariant. Naturality of the transverse invariants is slightly subtle. We note that Baldwin, Vela-Vick, and Vértesi [BVVV13, Theorem 3.1] defined the BRAID invariant only up to an automorphism of knot Floer homology, as their transition maps change the embedding of the link, as well as the number of basepoints. We now sketch a slightly stronger naturality result.

Proposition 8.6. Suppose that $\mathbb{L}$ is a multi-pointed, transverse link in the contact 3-manifold $(Y, \xi)$. If $\mathcal{O}$ and $\mathcal{O}^{\prime}$ are two pointed, embedded open books compatible with $(Y, \mathbb{L}, \xi)$, then

$$
\Psi_{\mathcal{H}^{\vee}(\mathcal{O}) \rightarrow \mathcal{H}^{\vee}\left(\mathcal{O}^{\prime}\right)}([\mathbf{x}(\mathcal{O})])=\left[\mathbf{x}\left(\mathcal{O}^{\prime}\right)\right],
$$

where $\Psi_{\mathcal{H}^{\vee}(\mathcal{O}) \rightarrow \mathcal{H}^{\vee}\left(\mathcal{O}^{\prime}\right)}$ denotes the map from naturality [JTZ], and $\mathbf{x}(\mathcal{O})$ and $\mathbf{x}\left(\mathcal{O}^{\prime}\right)$ are the canonical intersection points representing the transverse invariant. 

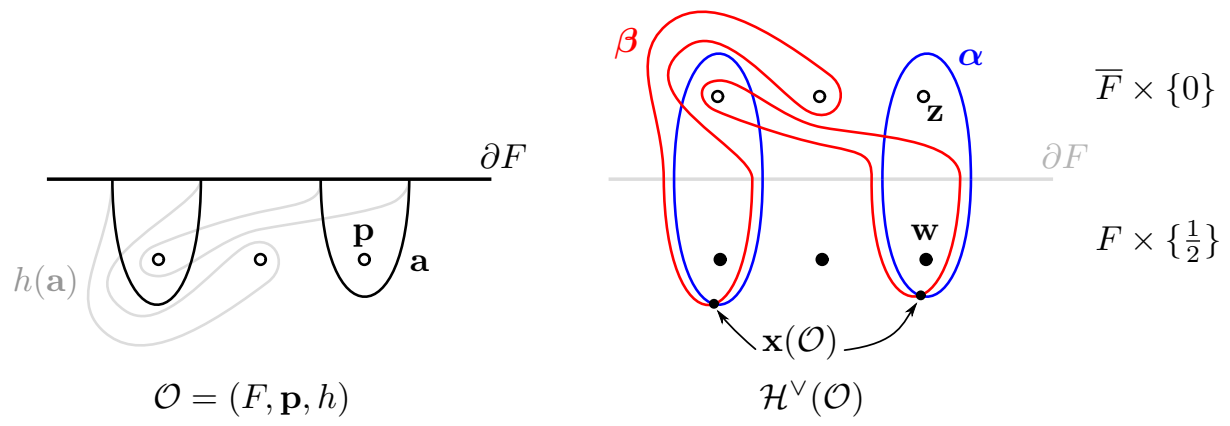

Figure 8.2. A pointed open book $\mathcal{O}$ (left), and the Heegaard diagram $\mathcal{H}^{\vee}(\mathcal{O})$ of $(Y, \mathbb{L})$ (right). The intersection point $\mathbf{x}(\mathcal{O})$ is shown.

Proof. The key ingredients for the proof of Proposition 8.6 that differ from the standard proofs of the invariance of the contact class, are as follows. By Proposition 7.10, if $\mathcal{O}$ is a pointed open book compatible with $(Y, \mathbb{L}, \xi)$, then the partial open book $\mathcal{O}^{\circ}$ described in Section 7.4 is adapted to the contact structure $\xi_{\mathbb{L}}^{\dagger}$ on the sutured manifold $Y(\mathbb{L})$. Furthermore, it is not hard to see that any partial open book for $\left(Y(\mathbb{L}), \xi_{\mathbb{L}}^{\dagger}\right)$ may be used to compute the transverse invariants (even the minus versions). Hence, Proposition 8.6 is proven by following the proof of the naturality of the sutured contact class of Honda, Kazez, and Matić [HKM09] using the relative Giroux correspondence for partial open books, applied to $\left(Y(\mathbb{L}), \xi_{\mathbb{L}}^{\pitchfork}\right)$.

8.3. Transverse invariants and positive saddle cobordisms. In this section, we show that the dual of the link cobordism map for a positive saddle preserves the transverse invariant. We need the following definition:

Definition 8.7. Suppose that $(W, \mathcal{S}):\left(Y, \mathbb{L}_{0}\right) \rightarrow\left(Y, \mathbb{L}_{1}\right)$ is a decorated link cobordism, where $W=[a, b] \times Y$ and $\mathcal{S}=(S, \mathcal{A})$. We say that $(W, \mathcal{S})$ is a standard $\mathbf{z}$-saddle cobordism if the following hold:

(1) The projection $\pi:[a, b] \times Y \rightarrow[a, b]$ restricts to a Morse function on $S$, with a single index 1 critical point.

(2) The index 1 critical point of $\left.\pi\right|_{S}$ occurs in the subregion $S_{\mathbf{z}} \subseteq S \backslash \mathcal{A}$.

(3) The restriction $\left.\pi\right|_{\mathcal{A}}$ has no critical points.

A standard $\mathbf{w}$-saddle cobordism is defined similarly.

Lemma 8.8. Suppose that $(W, \mathcal{S}):\left(Y, \mathbb{L}_{0}, \xi\right) \rightarrow\left(Y, \mathbb{L}_{1}, \xi\right)$ is an ascending link cobordism with $W=$ $[a, b] \times Y \subseteq \operatorname{Symp}(Y, \xi)$, which is a standard $\mathbf{z}$-saddle cobordism, whose critical point is positive. Then

for $\circ \in\{\wedge,-\}$.

$$
\left(F_{W, \mathcal{S}}^{\circ}\right)^{\vee}\left(\mathfrak{T}^{\circ}\left(\mathbb{L}_{1}\right)\right)=\mathfrak{T}^{\circ}\left(\mathbb{L}_{0}\right),
$$

Proof. By Lemma 7.12, if $\mathbb{L}_{0}$ and $\mathbb{L}_{1}$ are transverse links in $(Y, \xi)$, and $\mathbb{L}_{1}$ differs from $\mathbb{L}_{0}$ by a positive saddle attachment, then we may pick embedded, pointed open books

$$
\mathcal{O}_{0}=\left(F, h_{0}, \mathbf{p}\right) \quad \text { and } \quad \mathcal{O}_{1}=\left(F, h_{1}, \mathbf{p}\right)
$$

for $\left(Y, \mathbb{L}_{0}, \xi\right)$ and $\left(Y, \mathbb{L}_{1}, \xi\right)$, respectively, such that $h_{1}=\mathrm{tw}_{\gamma}^{+} \circ h_{0}$ for some path $\gamma$ connecting two basepoints $p_{1}, p_{2} \in \mathbf{p}$, where $\mathrm{tw}_{\gamma}^{+}$denotes a positive half-twist along $\gamma$.

Pick a basis of $\operatorname{arcs} \mathbf{a}=\left(a_{1}, \ldots, a_{n}\right)$ of $F$ such that there is exactly one arc, $a_{i}$, such that $h\left(a_{i}\right) \cap \gamma$ is non-empty, and furthermore,

$$
\left|h\left(a_{i}\right) \cap \gamma\right|=1 .
$$

Let $(\Sigma, \boldsymbol{\alpha}, \boldsymbol{\beta}, \mathbf{w}, \mathbf{z})$ and $\left(\Sigma, \boldsymbol{\alpha}, \boldsymbol{\beta}^{\prime}, \mathbf{w}, \mathbf{z}\right)$ be the multi-pointed link diagrams for $\left(Y, \mathbb{L}_{0}\right)$ and $\left(Y, \mathbb{L}_{1}\right)$ constructed from the pointed open books $\mathcal{O}_{0}$ and $\mathcal{O}_{1}$ and the arc basis a, respectively, as described in 
Section 8.1. Consider the triple diagram $\left(\Sigma, \boldsymbol{\alpha}, \boldsymbol{\beta}, \boldsymbol{\beta}^{\prime}, \mathbf{w}, \mathbf{z}\right)$, where $\boldsymbol{\alpha}, \boldsymbol{\beta}$, and $\boldsymbol{\beta}^{\prime}$ intersect on $F \times\left\{\frac{1}{2}\right\}$ as shown on the right of Figure 8.3.

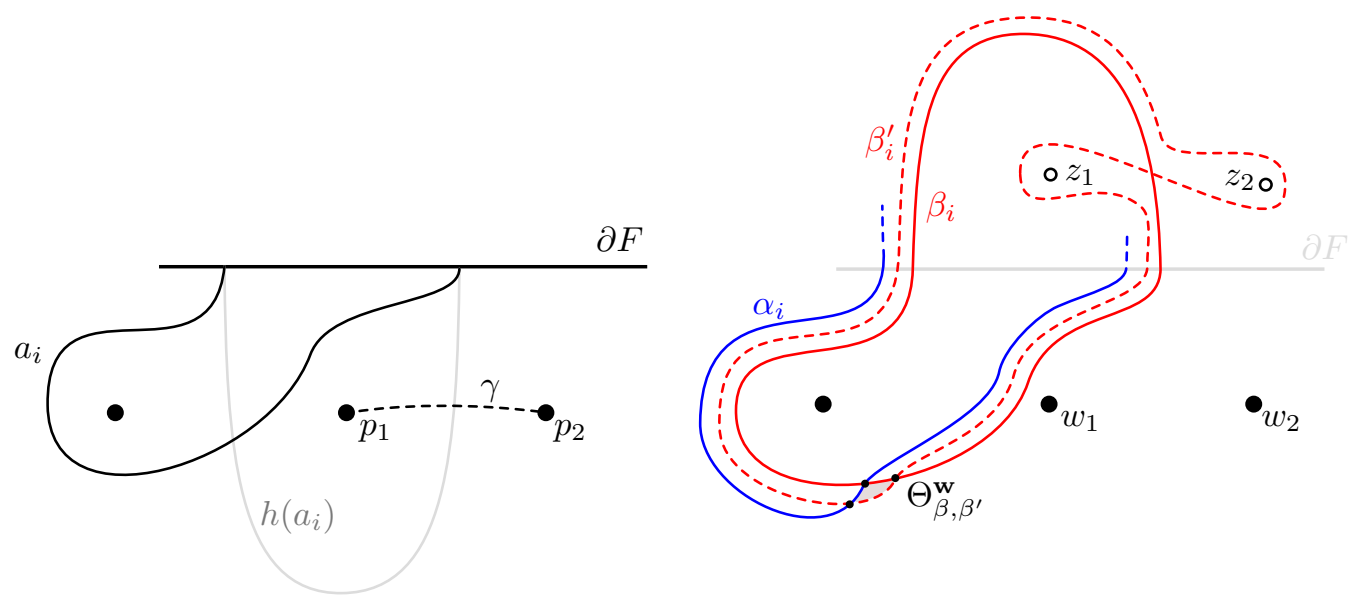

Figure 8.3. This model computation shows that the reverse of a positive saddle cobordism preserves $\mathfrak{T}^{\circ}$. Note that the top half of the right-hand side represents $\bar{F} \times\{0\}$. Hence a positive half-twist on $F$ appears as a negative one on $\bar{F} \times\{0\}$.

Let $B \subseteq Y$ denote the band for the link $L_{0}$, obtained by projecting the saddle cobordism into $Y$. In terms of [Zem19b, Definition 6.2], the triple $\left(\Sigma, \boldsymbol{\alpha}, \boldsymbol{\beta}, \boldsymbol{\beta}^{\prime}, \mathbf{w}, \mathbf{z}\right)$ is subordinate to the band $B$. Consequently, we may use this triple to compute the link cobordism maps for the saddle. Similarly, the triple $\left(\Sigma, \boldsymbol{\beta}, \boldsymbol{\beta}^{\prime}, \boldsymbol{\alpha}, \mathbf{w}, \mathbf{z}\right)$ is subordinate to the reversed band $B^{\vee}$, now viewed as being attached to $\mathbb{L}_{1}$ to form $\mathbb{L}_{0}$.

There are two canonical intersection points

$$
\Theta_{\beta, \beta^{\prime}}^{\mathbf{w}}, \Theta_{\beta, \beta^{\prime}}^{\mathbf{z}} \in \mathbb{T}_{\beta} \cap \mathbb{T}_{\beta^{\prime}},
$$

distinguished by their multi-gradings. The element $\Theta_{\beta, \beta^{\prime}}^{\mathbf{w}}$ is the top $\operatorname{gr}_{\mathbf{w}}$-graded intersection point of $\mathbb{T}_{\beta} \cap \mathbb{T}_{\beta^{\prime}}$, while $\Theta_{\beta, \beta^{\prime}}^{\mathbf{z}}$ is the top gr $_{\mathbf{z}}$-graded intersection point. The type-w and type-z saddle maps are defined via the triangle counts

$$
F_{B^{\vee}}^{\mathbf{w}}(-):=F_{\beta, \beta^{\prime}, \alpha}\left(\Theta_{\beta, \beta^{\prime}}^{\mathbf{z}} \otimes-\right) \quad \text { and } \quad F_{B^{\vee}}^{\mathbf{z}}(-):=F_{\beta, \beta^{\prime}, \alpha}\left(\Theta_{\beta, \beta^{\prime}}^{\mathbf{w}} \otimes-\right) .
$$

The map $F_{B \vee}^{\mathbf{w}}$ in equation (25) is the cobordism map for a standard $\mathbf{w}$-saddle cobordism, and the $F_{B^{\vee}}^{\mathbf{z}}$ is the map for the cobordism map for a standard $\mathbf{z}$-saddle cobordism. It is straightforward to see that

$$
F_{B^{\vee}}^{\mathbf{z}}=\left(F_{B}^{\mathbf{z}}\right)^{\vee},
$$

as a special case of equation (2).

For the main claim, it is sufficient to prove

$$
F_{B^{\vee}}^{\mathbf{z}}\left(\mathfrak{T}^{\circ}\left(\mathcal{O}_{1}\right)\right)=\mathfrak{T}^{\circ}\left(\mathcal{O}_{0}\right) .
$$

Equation (26) is verified via the model computation shown in Figure 8.3, since there is a unique holomorphic triangle with corners at $\Theta_{\beta, \beta^{\prime}}^{\mathbf{w}}$ and $\mathbf{x}\left(\mathcal{O}_{1}\right)$ that has zero multiplicity at the $\mathbf{w}$ basepoints. Furthermore, this triangle has index 0 , and has third corner at $\mathbf{x}\left(\mathcal{O}_{0}\right)$. The proof is complete.

\subsection{Transverse invariants and positive birth cobordisms.}

Lemma 8.9. Suppose that $\mathbb{L}$ is a multi-pointed, transverse link in the contact 3-manifold $(Y, \xi)$, and $\mathbb{U}$ is a doubly pointed, transverse unknot in $Y$, which is unlinked from $\mathbb{L}$. Suppose that

$$
(W, \mathcal{S}):(Y, \mathbb{L}, \xi) \rightarrow(Y, \mathbb{L} \cup \mathbb{U}, \xi)
$$


is an ascending link cobordism in $W=[a, b] \times Y \subseteq \operatorname{Symp}(Y, \xi)$ such that $\mathcal{S}$ is equal to $L \times I \sqcup \mathcal{D}_{0}$, where $\mathcal{D}_{0}$ is a positive birth. Furthermore, assume the dividing set on $\mathcal{D}_{0}$ consists of a single arc and is I-invariant on $L \times I$. Then

$$
\left(F_{W, \mathcal{S}}^{\circ}\right)^{\vee}\left(\mathfrak{T}^{\circ}(\mathbb{L} \cup \mathbb{U})\right)=\mathfrak{T}^{\circ}(\mathbb{L}),
$$

for $\circ \in\{\wedge,-\}$.

Proof. By Lemma 7.11, we may construct pointed open books $\mathcal{O}$ and $\mathcal{O}^{\prime}$ for $\mathbb{L}$ and $\mathbb{L} \cup \mathbb{U}$, respectively, such that $\mathcal{O}=(F, h, \mathbf{p})$ and $\mathcal{O}^{\prime}=\left(F, h, \mathbf{p} \cup\left\{p^{\prime}\right\}\right)$, where $p^{\prime}$ is a new basepoint which is close to $\partial F$; see Figure 8.4.

There is a chain isomorphism

$$
C F L^{\circ}\left(\mathcal{H}^{\vee}\left(\mathcal{O}^{\prime}\right)\right) \cong C F L^{\circ}\left(\mathcal{H}^{\vee}(\mathcal{O})\right) \otimes\left\langle\theta^{-}, \theta^{+}\right\rangle,
$$

where $\theta^{-}$and $\theta^{+}$are distinguished by the Maslov grading. By construction,

$$
\mathfrak{T}^{\circ}\left(\mathcal{O}^{\prime}\right)=\mathfrak{T}^{\circ}(\mathcal{O}) \times \theta^{-} .
$$

The map $\left(F_{W, \mathcal{S}}^{\circ}\right)^{\vee}$ coincides with the map for a death cobordism, which is given by the formula

$$
\left(F_{W, \mathcal{S}}^{\circ}\right)^{\vee}(\mathbf{x} \times \theta)= \begin{cases}\mathbf{x} & \text { if } \theta=\theta^{-}, \\ 0 & \text { if } \theta=\theta^{+},\end{cases}
$$

extended equivariantly over the action of $v$, if $\circ=-$. The result immediately follows.
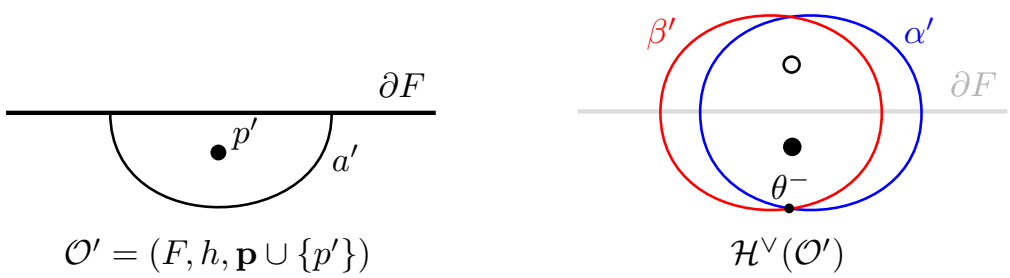

Figure 8.4. Left: Adding a new point $p^{\prime}$ and an $\operatorname{arc} a^{\prime}$ to an open book near $\partial F$, as in Lemma 8.9. Right: The resulting Heegaard diagram.

8.5. Transverse invariants and quasi-stabilizations. In this section, we prove that the transverse invariant is functorial with respect to certain simple decorations on cylindrical link cobordisms. Our proof is a repackaging of Baldwin, Vela-Vick and Vértesi's proof that the BRAID invariant is invariant under positive Markov stabilizations [BVVV13, p. 948].

We recall the TQFT framework of the third author for adding and removing basepoints from a link, as described in [Zem17] and [Zem19b]. Suppose that $(L, \mathbf{w}, \mathbf{z})$ is a multi-pointed link in $Y$, and $(w, z)$ is a new pair of basepoints on $L$, which are in the same component of $L \backslash(\mathbf{w} \cup \mathbf{z})$. Suppose, for definiteness, that $w$ follows $z$ with respect to the orientation of $L$. The third author described chain maps

$$
\begin{aligned}
& S_{w, z}^{+}, T_{w, z}^{+}: C F L^{\circ}(Y, L, \mathbf{w}, \mathbf{z}) \rightarrow C F L^{\circ}(Y, L, \mathbf{w} \cup\{w\}, \mathbf{z} \cup\{z\}) \text { and } \\
& S_{w, z}^{-}, T_{w, z}^{-}: C F L^{\circ}(Y, L, \mathbf{w} \cup\{w\}, \mathbf{z} \cup\{z\}) \rightarrow C F L^{\circ}(Y, L, \mathbf{w}, \mathbf{z}) .
\end{aligned}
$$

The maps $S_{w, z}^{ \pm}$and $T_{w, z}^{ \pm}$correspond to special dividing sets on the cylinder $I \times L$, as shown in Figure 8.5. Note that any dividing set on $I \times L$ can be obtained, up to isotopy, by composing such dividing sets.

The maps $S_{w, z}^{+}$and $T_{w, z}^{+}$are obtained by stabilizing the Heegaard diagram in a special way; see Figure 8.6. This operation is called a linked index 0/3 stabilization in [BVVV13], and a quasistabilization in [MO10]. The third author showed that the operation is natural [Zem17, Theorem A], and gave a link cobordism interpretation [Zem19b, Section 4] using the decorated cobordisms in Figure 8.5. 

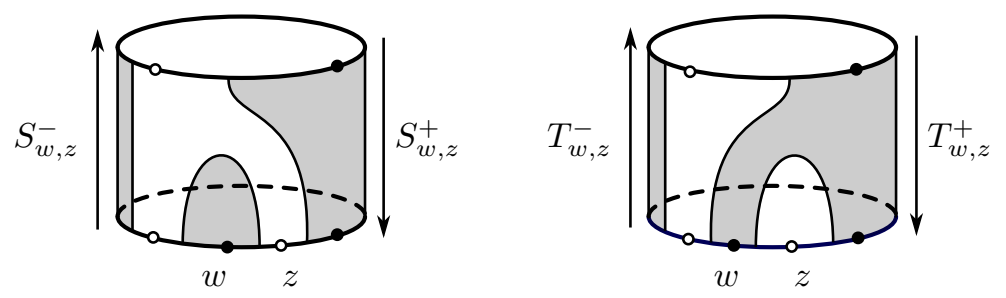

FiguRE 8.5. Dividing sets for the maps $T_{w, z}^{+}, T_{w, z}^{-}, S_{w, z}^{+}$, and $S_{w, z}^{-}$.

Lemma 8.10. Suppose that $\mathbb{L}=(L, \mathbf{w}, \mathbf{z})$ is a multi-pointed, transverse link in $(Y, \xi)$, and $\mathbb{L}^{\prime}$ is obtained by adding two new, adjacent basepoints $w$ and $z$ to $\mathbb{L}$. For $\circ \in\{\wedge,-\}$, we have

$$
\left(S_{w, z}^{+}\right)^{\vee}\left(\mathfrak{T}^{\circ}\left(Y, \mathbb{L}^{\prime}, \xi\right)\right)=\mathfrak{T}^{\circ}(Y, \mathbb{L}, \xi) \quad \text { and } \quad\left(T_{w, z}^{-}\right)^{\vee}\left(\mathfrak{T}^{\circ}(Y, \mathbb{L}, \xi)\right)=\mathfrak{T}^{\circ}\left(Y, \mathbb{L}^{\prime}, \xi\right) \text {. }
$$

Proof. Our proof follows by analyzing Baldwin, Vela-Vick, and Vértesi's proof of the invariance of the BRAID invariant under positive Markov stabilizations; see [BVVV13, Figure 11]. We pick a pointed open book $\mathcal{O}=(F, h, \mathbf{p})$ for $(Y, \mathbb{L}, \xi)$. We may construct a pointed open book $\mathcal{O}^{\prime}=\left(F, h^{\prime}, \mathbf{p}^{\prime}\right)$ for $\left(Y, \mathbb{L}^{\prime}, \xi\right)$, as follows. We define $\mathbf{p}^{\prime}=\mathbf{p} \cup\{p\}$, where $p$ is a point near $\partial F$. We pick a path $\gamma$ from $p$ to another point $p^{\prime} \in \mathbf{p}$, and set $h^{\prime}$ to be $\mathrm{tw}_{\gamma}^{+} \circ h$. We pick a basis of arcs a for $\mathcal{O}$ such that $p$ and $p^{\prime}$ lie in the same component of $F \backslash \mathbf{a}$, and pick a basis $\mathbf{a}^{\prime}$ for $\mathcal{O}^{\prime}$ by adjoining an arc $a$ that bounds a bigon with $\partial F$ containing $p$.
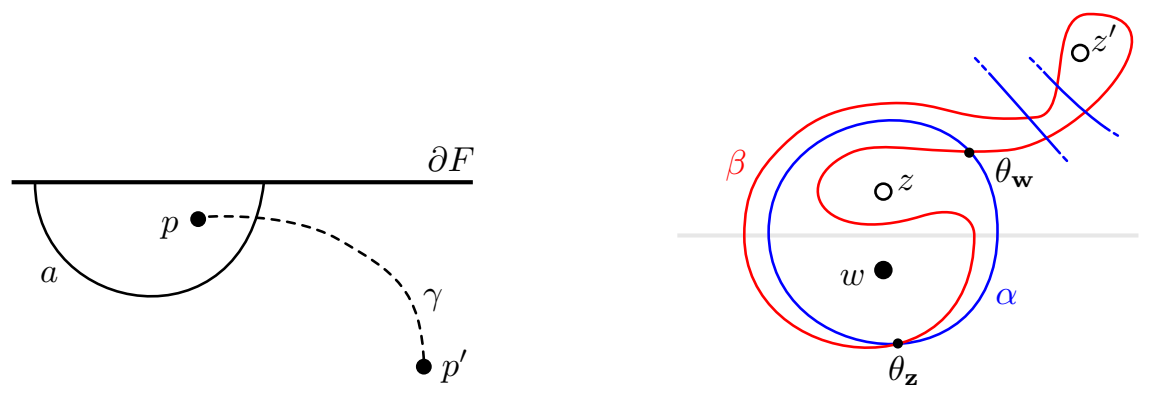

FiguRE 8.6. Left: The effect of a positive Markov stabilization on the open book $\mathcal{O}$. Right: Its effect on the Heegaard diagram $\mathcal{H}^{\vee}(\mathcal{O})$.

The Heegaard diagram $\mathcal{H}\left(\mathcal{O}^{\prime}\right)$ is shown in Figure 8.6. There is an isomorphism of chain complexes

$$
C F L^{\circ}\left(\mathcal{H}^{\vee}\left(\mathcal{O}^{\prime}\right)\right) \cong C F L^{\circ}\left(\mathcal{H}^{\vee}(\mathcal{O})\right) \otimes\left\langle\theta_{\mathbf{w}}, \theta_{\mathbf{z}}\right\rangle,
$$

where $\theta_{\mathbf{w}}$ and $\theta_{\mathbf{z}}$ are the two intersection points labeled in Figure 8.6. The point $\theta_{\mathbf{w}}$ is the highergraded intersection point with respect to $\mathrm{gr}_{\mathbf{w}}$, and $\theta_{\mathbf{z}}$ is the higher-graded intersection point with respect to $\mathrm{gr}_{\mathbf{z}}$.

The maps $T_{w, z}^{ \pm}$and $S_{w, z}^{ \pm}$are defined via the formulas

$$
T_{w, z}^{+}(\mathbf{x})=\mathbf{x} \times \theta_{\mathbf{z}} \quad \text { and } \quad T_{w, z}^{-}(\mathbf{x} \times \theta)= \begin{cases}\mathbf{x} & \text { if } \theta=\theta_{\mathbf{w}} \\ 0 & \text { if } \theta=\theta_{\mathbf{z}}\end{cases}
$$

and

Note that

$$
S_{w, z}^{+}(\mathbf{x})=\mathbf{x} \times \theta_{\mathbf{w}} \quad \text { and } \quad S_{w, z}^{-}(\mathbf{x} \times \theta)= \begin{cases}0 & \text { if } \theta=\theta_{\mathbf{w}} \\ \mathbf{x} & \text { if } \theta=\theta_{\mathbf{z}}\end{cases}
$$

Hence, we obtain that

$$
\mathfrak{T}^{\circ}\left(\mathcal{O}^{\prime}\right)=\mathfrak{T}^{\circ}(\mathcal{O}) \times \theta_{\mathbf{z}}
$$

$$
S_{w, z}^{-}\left(\mathfrak{T}^{\circ}\left(Y, \mathbb{L}^{\prime}, \xi\right)\right)=\mathfrak{T}^{\circ}(Y, \mathbb{L}, \xi) \quad \text { and } \quad T_{w, z}^{+}\left(\mathfrak{T}^{\circ}(Y, \mathbb{L}, \xi)\right)=\mathfrak{T}^{\circ}\left(Y, \mathbb{L}^{\prime}, \xi\right) .
$$


The main result now follows from equation (27) by noting that $S_{w, z}^{+}$is dual to $S_{w, z}^{-}$, and $T_{w, z}^{+}$is dual to $T_{w, z}^{-}$.

8.6. Proof of Theorem 1.2. In this section, we prove Theorem 1.2. The following terminology will be convenient:

Definition 8.11. Suppose that $\mathcal{S}=(S, \mathcal{A})$ is a decorated surface, equipped with a Morse function $\phi$ such that $\left.\phi\right|_{\mathcal{A}}$ is also Morse. We say that a critical point $p$ of $\left.\phi\right|_{\mathcal{A}}$ is of type $S^{+}$if $\left.\phi\right|_{\mathcal{A}}$ has a local minimum at $p$, and the region $\mathcal{S}_{\mathbf{w}}$ lies immediately above $p$, and $\mathcal{S}_{\mathbf{z}}$ lies immediately below, as in the reverse of the left-hand side of Figure 8.5. A critical point is similarly said to be of type $S^{-}$, $T^{+}$, or $T^{-}$if it satisfies the analogous configuration shown in Figure 8.5.

We now reformulate the definition of $\mathbf{w}$-arboreal decorations.

Lemma 8.12. Suppose that that $\mathcal{S}=(S, \mathcal{A})$ is a surface with divides, with $\partial S=-L_{0} \cup L_{1}$. Suppose $\phi: S \rightarrow[0,1]$ is a Morse function, and $\phi$ has only index 1 and 2 critical points. Then $\mathcal{S}$ is $\mathbf{w}$-arboreal with respect to $\phi$ if and only if the dividing set $\mathcal{A}$ may be isotoped so that the following hold:

(1) All index 2 critical points of $\phi$ occur along $\mathcal{A}$.

(2) All index 1 critical points of $\phi$ occur in $\mathcal{S}_{\mathbf{z}}$.

(3) The function $\left.\phi\right|_{\mathcal{A}}$ is Morse, with only type $S^{-}$and $T^{+}$critical points.

Proof. Suppose first $\mathcal{S}$ is $\mathbf{w}$-arboreal with respect to $\phi$, and let $F$ be the forest in the definition. Perturbing $F$ slightly, we may assume that $F$ is disjoint from the critical set of $\phi$. Next, we add an edge to $F$ for each index 2 critical point, by flowing from each critical point downward along the gradient of $\phi$, until it hits either an edge of $F$ or $L_{0}$. If it hits $F$, we are done. If the downward trajectory hits $L_{0}$, we then isotope it near $L_{0}$ so that it intersects an edge of $F$. Let $\mathcal{A}=\operatorname{cl}(N(F) \backslash \partial S)$, for a suitably chosen regular neighborhood $N(F)$ of $F$. After moving $\mathcal{A}$ very slightly near the index 2 critical points, so they lie on $\mathcal{A}$, it is straightforward to check that it has the stated properties.

Conversely, suppose that $\mathcal{S}$ satisfies the properties (1), (2), (3). We may decompose $\mathcal{S}$ into a composition of standard $\mathbf{w}$-saddles, deaths occurring along the dividing set, as well as cylindrical link cobordisms where $\left.\phi\right|_{\mathcal{A}}$ has a single critical point, which further has the configuration of type $T^{+}$or $S^{-}$. Since the $\mathbf{w}$-arboreal property is preserved by composition, it is sufficient to check the w-arboreal condition for each of the above elementary cobordisms, which is straightforward.

Proof of Theorem 1.2. According to the work of Eliashberg [Eli90] and Gompf [Gom98], the Morse function $\phi$ has critical points only of index 0,1 , and 2. Passing an index 1 critical point of $\phi$ has the effect of removing two standard Darboux balls, and gluing a standard $S^{2} \times I$ along two convex copies of $S^{2}$. The 2-handles are attached along contact $(-1)$-framed Legendrians. Furthermore, if $W$ is connected and $Y_{0}$ is non-empty, then we may assume that there are no 0-handles.

By Lemma 7.5, $\left.\phi\right|_{S}$ has only index 0 and 1 critical points. By Lemma 8.12, after an isotopy of the decoration, we may assume that the dividing set intersects any index 0 critical point of $\left.\phi\right|_{S}$, all index 1 critical points of $\left.\phi\right|_{S}$ occur in $\mathcal{S}_{\mathbf{z}}$, and also that every critical point of $\left.\phi\right|_{\mathcal{A}}$ is of type $S^{+}$or $T^{-}$. It is sufficient to prove the case when $(W, \mathcal{S})$ is an ascending link cobordism, with w-anti-arboreal decoration, such that $(W, \mathcal{S})$ contains at most 1 critical point of one of the functions $\phi,\left.\phi\right|_{\mathcal{S}}$, or $\left.\phi\right|_{\mathcal{A}}$.

First, if $\phi,\left.\phi\right|_{\mathcal{S}}$, and $\left.\phi\right|_{\mathcal{A}}$ have no critical points, and $\partial W=-Y_{0} \cup Y_{1}$, with $\phi\left(Y_{i}\right)=i$, then there is a diffeomorphism between $W$ and $I \times Y$ that intertwines $\phi$ with the projection onto the first factor, and intertwines the contact structure $i_{V} \omega$ on $\phi^{-1}(c)$ with some fixed contact structure $\xi$ on $Y$, for all $c \in I$; see [Hay17, Lemma 4.12]. This diffeomorphism maps $S$ to the trace of a transverse isotopy of links in $(Y, \xi)$. A straightforward Moser-type argument implies that $S$ is the trace of a contact isotopy of $(Y, \xi)$. Furthermore, this isotopy may be assumed to be compatible with the dividing set. Hence, functoriality of $\mathfrak{T}^{\circ}$, for this cobordism, follows from the naturality of $\mathfrak{T}^{\circ}$, Proposition 8.6.

The proof of the statement for Weinstein 1-handles and 2-handles adapts immediately from the proof given by Ozsváth and Szabó [OS05] for functoriality of the contact invariant of closed 3manifolds with respect to Stein cobordisms, as in [Juh16, Theorem 11.24]. See [OS05, Theorem 4.2] 
for the proof of the statement about Weinstein 2-handles. For Weinstein 1-handles, the computation is as follows. If $\mathcal{O}=(S, P, h)$ is an open book for $\left(Y_{0}, \mathbb{L}_{0}\right)$, and $\left(Y_{1}, \mathbb{L}_{1}\right)$ is obtained by adding a Weinstein 1-handle, then we may obtain an open book for $\left(Y_{1}, \mathbb{L}_{1}\right)$, by attaching a band to $\partial S$, and extending $h$ via the identity over the band. A straightforward computation verifies the claim in this case.

It remains to verify the statement for cobordisms which have a single critical point of $\left.\phi\right|_{S}$ or a critical point of $\left.\phi\right|_{\mathcal{A}}$. The claim for index 0 critical points of $\left.\phi\right|_{S}$ is Lemma 8.9. The claim for index 1 critical points of $\left.\phi\right|_{S}$ is Lemma 8.8. Finally, the claim for critical points of $\left.\phi\right|_{\mathcal{A}}$ is Lemma 8.10. The proof is complete.

We now sketch the proof of the perturbed version:

Proof of Proposition 8.5. The proof is similar to the proof of Theorem 1.2. We decompose the cobordism $(W, \mathcal{S})$ into a sequence of positive births, positive saddles, $S^{+}$and $T^{-}$decorations, and Weinstein 1- and 2-handles. In between the moves, we may also have to perform open book isotopies, and Giroux stabilizations of the open books.

The key observation is that, for each of the associated maps, if $\mathcal{O}_{0}$ is an open book for the incoming end, and $\mathcal{O}_{1}$ is an open book for the outgoing end, then the dual of the cobordism or naturality map sends the canonical intersection point $\mathbf{x}\left(\mathcal{O}_{1}\right) \otimes 1$ to $\mathbf{x}\left(\mathcal{O}_{0}\right) \otimes e^{\left(a_{1}, \ldots, a_{n}\right)}$. For example, the dual of the cobordism map for a positive saddle counts exactly one homotopy class of triangles when applied to $\mathbf{x}\left(\mathcal{O}_{1}\right)$, as illustrated in Figure 8.3. The other types of elementary ascending and Weinstein cobordisms are analyzed similarly.

\section{Proof of Theorem 1.1}

We are now ready to prove Theorem 1.1 from the introduction.

Proof of Theorem 1.1. According to Rudolph [Rud93, Lemma 2], the untwisted positive Whitehead double of a nontrivial strongly quasipositive knot is strongly quasipositive. Furthermore, the genus one Seifert surface obtained by taking an untwisted annulus about the knot, and plumbing it with $\mathrm{a}+1$ twisted annulus about the unknot is a quasipositive Seifert surface for the Whitehead double.

Let $K_{0}$ be a non-trivial strongly quasipositive knot in $S^{3}$, write $K_{1}$ for its untwisted positive Whitehead double, and let $K$ be the untwisted positive Whitehead double of $K_{1}$. Then both $K_{1}$ and $K$ are strongly quasipositive. Furthermore, they are topologically slice by the work of Freedman [FQ90], as they have Alexander polynomial one. Let $S$ be the standard genus one Seifert surface of $K$, obtained by plumbing an untwisted annulus about $K_{1}$ with a +1 twisted annulus about the unknot. In particular, $\gamma:=K_{1}$ lies on the surface $S$, and the surface framing of $\gamma$ is trivial. By construction, $\gamma$ is topologically slice, so it bounds a topological disk in the complement of $S$. Furthermore, $\gamma$ is homologically nontrivial in $S$.

The pair $(S, \gamma)$ satisfies the assumptions of Theorem 2.5. For every $n \in \mathbb{Z}$, choose a knot $J_{n}$ such that its Alexander polynomial $\Delta_{J_{n}}(z)$ has $n$ irreducible factors. For example, suppose that $J$ is a nontrivial knot such that $\Delta_{J}(z)$ is irreducible, and take $J_{n}:=\#_{n} J$. Let $C_{n}:=\left(J_{n}\right)_{\text {tw }}$ be the 1-twist self-concordance of $J_{n}$, as in Definition 2.3. As the automorphism $\phi$ of $S^{3}$ used in the construction of $C_{n}$ is isotopic to id $S^{3}$ through automorphisms of $S^{3}$ that fix $J_{n}$ as a based knot pointwise throughout, $\phi$ induces the identity on $\widehat{H F K}\left(J_{n}\right)$, and so $\operatorname{Lef}_{z}\left(C_{n}\right)=\Delta_{J_{n}}(z)$. Finally, $F_{B^{4}, S} \neq 0$ by Corollary 8.3, as $S$ is strongly quasipositive. It follows from Theorem 6.6 that the 1-twist rim surgered surfaces $S\left(\gamma, C_{n}\right)$ are pairwise non-diffeomorphic.

\section{REFERENCES}

[Akb01] Selman Akbulut, Variations on Fintushel-Stern knot surgery on 4-manifolds, Proceedings of 8th Gökova Geometry-Topology conference, 2001, pp. 81-92.

[BLW19] John A. Baldwin, Tye Lidman, and C.-M. Michael Wong, Lagrangian cobordisms and Legendrian invariants in knot Floer homology, 2019. e-print, arXiv:1907.09654.

[BO01] Michel Boileau and Stepan Orevkov, Quasi-positivité d'une courbe analytique dans une boule pseudoconvexe, C. R. Acad. Sci. Paris Sér. I Math. 332 (2001), no. 9, 825-830. MR1836094 
[BS16] John Baldwin and Steven Sivek, On the equivalence of contact invariants in sutured Floer homology theories, 2016. e-print, arXiv:1601.04973.

[BS18] John A. Baldwin and Steven Sivek, Invariants of Legendrian and transverse knots in monopole knot homology, J. Symplectic Geom. 16 (2018), no. 4, 959-1000. MR3917725

[BVVV13] John A. Baldwin, David Shea Vela-Vick, and Vera Vértesi, On the equivalence of Legendrian and transverse invariants in knot Floer homology, Geom. Topol. 17 (2013), no. 2, 925-974. MR3070518

[CE12] Kai Cieliebak and Yakov Eliashberg, From Stein to Weinstein and back, American Mathematical Society Colloquium Publications, vol. 59, American Mathematical Society, Providence, RI, 2012. Symplectic geometry of affine complex manifolds. MR3012475

[EFM01] Judith Epstein, Dmitry Fuchs, and Maike Meyer, Chekanov-Eliashberg invariants and transverse approximations of Legendrian knots, Pacific J. Math. 201 (2001), no. 1, 89-106. MR1867893

[EH01] John B. Etnyre and Ko Honda, Knots and contact geometry. I. Torus knots and the figure eight knot, J. Symplectic Geom. 1 (2001), no. 1, 63-120. MR1959579

[Eli90] Yakov Eliashberg, Topological characterization of Stein manifolds of dimension > 2, Internat. J. Math. 1 (1990), no. 1, 29-46. MR1044658

[Fin02] Sergey Finashin, Knotting of algebraic curves in $\mathbb{C} P^{2}$, Topology 41 (2002), no. 1, 47-55. MR1871240

[FKV87] S. M. Finashin, M. Kreck, and O. Ya. Viro, Exotic knottings of surfaces in the 4-sphere, Bull. Amer. Math. Soc. (N.S.) 17 (1987), no. 2, 287-290. MR903734

[FQ90] Michael Freedman and Frank Quinn, Topology of 4-manifolds, Princeton mathematical series, vol. 39, Princeton University Press, 1990.

[FS97] Ronald Fintushel and Ronald J. Stern, Surfaces in 4-manifolds, Math. Res. Lett. 4 (1997), no. 6, $907-914$. MR1492129

[GJ19] Marco Golla and András Juhász, Functoriality of the EH class and the loss invariant under Lagrangian concordances, 2019. To appear, Algebraic \& Geometric Topology. Avalable at arXiv:1801.03716.

[Gom91] Robert E. Gompf, Nuclei of elliptic surfaces, Topology 30 (1991), no. 3, 479-511. MR1113691

[Gom98] _ _ Handlebody construction of Stein surfaces, Ann. of Math. (2) 148 (1998), no. 2, 619-693. MR1668563

[Hay17] Kyle Hayden, Quasipositive links and Stein surfaces, 2017. e-print, arXiv:1703.10150.

[HKM09] Ko Honda, William H. Kazez, and Gordana Matić, The contact invariant in sutured Floer homology, Invent. Math. 176 (2009), 637-676.

[JM17] Andás Juhász and Marco Marengon, Computing cobordism maps in link Floer homology and the reduced Khovanov TQFT, Sel. Math. New. Ser. (2017).

[JTZ] András Juhász, Dylan Thurston, and Ian Zemke, Naturality and mapping class groups in Heegaard Floer homology, Mem. Amer. Math. Soc., to appear.

[Juh06] András Juhász, Holomorphic discs and sutured manifolds, Algebr. Geom. Topol. 6 (2006), 1429-1457.

[Juh16] _ Cobordisms of sutured manifolds and the functoriality of link Floer homology, Adv. Math. 299 (2016), 940-1038.

[JZ18a] András Juhász and Ian Zemke, Concordance surgery and the Ozsváth-Szabó 4-manifold invariant (2018). e-print, arXiv: 1804.06221.

[JZ18b] András Juhász and Ian Zemke, Contact handles, duality, and sutured Floer homology, 2018. e-print, arXiv: 1803.04401

[Kan18a] Sungkyung Kang, $\mathbb{Z}_{2}$-equivariant Heegaard Floer cohomology of knots in $S^{3}$ as a strong Heegaard invariant, 2018. e-print, arXiv:1810.01919.

[Kan18b] _ A transverse link invariant from $\mathbb{Z}_{2}$-equivariant Heegaard Floer cohomology, 2018. e-print, arXiv: 1802.00351.

[Kim06a] Hee Jung Kim, Modifying surfaces in 4-manifolds by twist spinning, Geom. Topol. 10 (2006), $27-56$. MR2207789

[Kim06b] _ Modifying surfaces in 4-manifolds by twist spinning, Geom. Topol. 10 (2006), 27-56. MR2207789

[KR08a] Hee Jung Kim and Daniel Ruberman, Smooth surfaces with non-simply-connected complements, Algebr. Geom. Topol. 8 (2008), no. 4, 2263-2287. MR2465741

[KR08b] _ , Topological triviality of smoothly knotted surfaces in 4-manifolds, Trans. Amer. Math. Soc. 360 (2008), no. 11, 5869-5881. MR2425695

[Lit79] Rick Litherland, Deforming twist-spun knots, Trans. Amer. Math. Soc. 250 (1979), 311-331.

[LOSS09] Paolo Lisca, Peter Ozsváth, András I. Stipsicz, and Zoltán Szabó, Heegaard Floer invariants of Legendrian knots in contact three-manifolds, J. Eur. Math. Soc. (JEMS) 11 (2009), no. 6, 1307-1363. MR2557137

[MO10] Ciprian Manolescu and Peter S. Ozsváth, Heegaard Floer homology and surgeries on links, 2010. e-print, arXiv: 1011.1317.

[OS04] Peter Ozsváth and Zoltán Szabó, Holomorphic disks and knot invariants, Adv. Math. 186 (2004), no. 1, 58-116.

[OS05] _ Heegaard Floer homology and contact structures, Duke Math. J. 129 (2005), no. 1, 39-61.

[OS06] _ Holomorphic triangles and invariants for smooth four-manifolds, Adv. Math. 202 (2006), no. 2, 326-400. 
[OS08] , Holomorphic disks, link invariants and the multi-variable Alexander polynomial, Algebr. Geom. Topol. 8 (2008), no. 2, 615-692.

[OST08] Peter Ozsváth, Zoltán Szabó, and Dylan Thurston, Legendrian knots, transverse knots and combinatorial Floer homology, Geom. Topol. 12 (2008), no. 2, 941-980. MR2403802

[Rud83] Lee Rudolph, Algebraic functions and closed braids, Topology 22 (1983), no. 2, 191-202. MR683760

[Rud93] _ Quasipositivity as an obstruction to sliceness, Bull. Amer. Math. Soc. (N.S.) 29 (1993), no. 1, 51-59. MR1193540

[Rud99] _ Positive links are strongly quasipositive, Proceedings of the Kirbyfest (Berkeley, CA, 1998), 1999, pp. 555-562. MR1734423

[Sun15] Nathan S. Sunukjian, A note on knot surgery, J. Knot Theory Ramifications 24 (2015), no. 9, 1520003, 5. MR3403209

[SV09] András I. Stipsicz and Vera Vértesi, On invariants for Legendrian knots, Pacific J. Math. 239 (2009), no. 1, 157-177. MR2449016

[Ter94] Masakazu Teragaito, Twist-roll spun knots, Proc. Amer. Math. Soc. 122 (1994), 597-599.

[Zee65] Christopher Zeeman, Twisting spun knots, Trans. Amer. Math. Soc. 115 (1965), 471-495.

[Zem17] Ian Zemke, Quasistabilization and basepoint moving maps in link Floer homology, Algebr. Geom. Topol. 17 (2017), no. 6, 3461-3518. MR3709653

[Zem19a] _ Link cobordisms and absolute gradings on link Floer homology, Quantum Topol. 10 (2019), no. 2, 207-323. MR3950650

[Zem19b] _ Link cobordisms and functoriality in link Floer homology, J. Topol. 12 (2019), no. 1, 94-220. MR3905679

Mathematical Institute, University of Oxford, Andrew Wiles Building, Radcliffe Observatory QuarTER, WOODSTOCK ROAD, OXFORD, OX2 6GG, UK

Email address: juhasza@maths.ox.ac.uk

Department of Mathematics, Princeton University, Princeton, NJ 08544, USA

Email address: maggiem@math.princeton.edu

Department of Mathematics, Princeton University, Princeton, NJ 08544, USA

Email address: izemke@math.princeton.edu 